TITLE:

\title{
Physical origin of hydrophobicity studied in terms of cold denaturation of proteins: comparison between water and simple fluids.
}

\section{AUTHOR(S):}

Yoshidome, Takashi; Kinoshita, Masahiro

\section{CITATION:}

Yoshidome, Takashi ... [et al]. Physical origin of hydrophobicity studied in terms of cold denaturation of proteins: comparison between water and simple fluids.. Physical chemistry chemical physics : PCCP 2012, 14(42): 14554-14566

\section{ISSUE DATE:}

2012-08-31

URL:

http://hdl.handle.net/2433/178683

\section{RIGHT:}

(C) Royal Society of Chemistry 2012.; This is not the published version. Please cite only the published version.; この論文は出版社版でありませ ん。引用の際には出版社版をご確認ご利用ください。 


\title{
Physical origin of hydrophobicity studied in terms of cold denatura- tion of proteins: Comparison between water and simple fluids
}

\author{
Takashi Yoshidome and Masahiro Kinoshita*
}

\author{
Received Xth $X X X X X X X X X X 20 X X$, Accepted Xth $X X X X X X X X X 20 X X$ \\ First published on the web Xth $X X X X X X X X X X 200 X$ \\ DOI: 10.1039/b000000x
}

\begin{abstract}
A clue to the physical origin of the hydrophobicity is in the experimental observations manifesting that it is weakened at low temperatures. By considering a solvophobic model protein immersed in water and three species of simple solvents, we analyze the temperature dependences of the changes in free energy, energy, and entropy of the solvent upon protein unfolding. The angledependent and radial-symmetric integral equation theories and the morphometric approach are employed in the analysis. Each of the changes is decomposed into two terms which depend on the excluded volume and on the area and curvature of solventaccessible surface, respectively. The excluded-volume term of the entropy change is further decomposed into two components representing the protein-solvent pair correlation and the protein-solvent-solvent triplet and higher-order correlation, respectively. We show that water crowding in the system becomes more serious upon protein unfolding but this effect becomes weaker as the temperature is lowered. If the hydrophobicity originated from the water structuring near a nonpolar solute, it would be strengthened upon the temperature lowering. Among the three species of simple solvents, considerable weakening of the solvophobicity at low temperatures is observed only for the solvent where the particles interact through strongly attractive potential and the particle size is as small as that of water. Even in the case of this solvent, however, cold denaturation of a protein cannot be reproduced. It would be reproducible if the attractive potential was substantially enhanced, but such enhancement causes the appearance of the metastability limit for a single liquid phase.
\end{abstract}

\section{Introduction}

The hydrophobic effect at ambient temperature is powerful enough to drive a variety of self-assembly processes in aqueous environments such as micelle formation, protein folding and aggregation, receptor-ligand binding, and lipid membrane formation ${ }^{1-7}$. However, there is much experimental evidence showing that the hydrophobicity is weakened at low temperatures for small nonpolar solutes, amphiphilic molecules, and biomolecules like proteins. For example, upon the temperature lowering, the solubility of methane increases $3,5,6$, the critical micelle concentration becomes higher, the average size of micelles for nonionic amphiphilic molecules becomes smaller $^{8}$, most of the proteins unfold at $250 \sim 260 \mathrm{~K}^{9-11}$ (this unfolding is referred to as cold denaturation; yeast frataxin ${ }^{9}$ unfolds at the exceptionally high temperature, $\sim 280 \mathrm{~K}$ ), and protein aggregation is dissociated ${ }^{12}$. Despite the crucial importance of the hydrophobicity, its physical origin still remains rather ambiguous. A prevailing view is that the hydrophobicity originates from the inability of nonpolar solutes to participate in hydrogen bonds of water molecules and the resultant water structuring near the solutes giving rise to entropic loss ${ }^{1}$.

Institute of Advanced Energy, Kyoto University, Gokasho, Uji, Kyoto, 6110011, Japan. Fax: 8177438 4695; Tel: 81774 383503; E-mail: kinoshit@iae.kyoto-u.ac.jp.
If this was true, the hydrophobicity would be strengthened due to the enhanced hydrogen bonding when the temperature is lowered, which clearly conflicts with the experimental observations described above ${ }^{6}$. We believe that a clue to the physical origin of the hydrophobicity is in its weakening at low temperatures.

In a previous study ${ }^{6}$, we calculated hydration thermodynamic quantities (hydration free energy $\mu$, entropy $S_{V}$, and energy $U_{V}$ ) of small hard-sphere solutes using the angledependent integral equation theory ${ }^{6,13-16}$ combined with a multipolar water model ${ }^{13,14}$. The calculation was performed under the isochoric condition. The dependence of $\beta \mu$ on $T$ ( $\beta=1 /\left(k_{\mathrm{B}} T\right), k_{\mathrm{B}}$ is the Boltzmann constant, and $T$ is the absolute temperature) was discussed because the Ostwald coefficient $\exp (-\beta \mu)$ is a measure of the hydrophobicity. As the measure increases, the hydrophobicity becomes weaker. In what follows, we recapitulate the significant results obtained. When the number density of bulk water is taken to be that of real water along the saturation curve, $\beta \mu$ possesses the maximum value at $\sim 323 \mathrm{~K}$, which is consistent with the experimental result known for methane ${ }^{3,5,6}$. The measure becomes larger monotonically as $T$ decreases from ambient temperature. This temperature dependence of the measure arises primarily from that of the translational component of $S_{V}$. The orientational component of $S_{V}$ exhibits the opposite temperature 
dependence, which can be associated with the enhanced hydrophobicity at low temperatures and understood indisputably on the basis of the conventional picture ${ }^{1}$. The translational component is substantially larger than the orientational component. Here, the translational and orientational components represent the contributions from the translational and orientational freedoms of water molecules restricted by solute insertion, respectively. The proposition reached is the following: What is responsible for the hydrophobic effect is not the hydrogen-bonding property but the interplay of the exceptionally small molecular size and strongly attractive interaction of water ${ }^{6}$. However, the relation between this proposition and the physical origin of the hydrophobicity exhibiting the characteristic temperature dependence mentioned above is to be elucidated further.

It has been pointed out for spherical solutes that the behavior of a sufficiently large solute is qualitatively different from that of a small one ${ }^{4}$. The hydration free energy $\mu$ of the former at ambient pressure is scaled by the water-accessible surface area (ASA) $A$ as expressed by

$$
\mu \simeq \gamma A
$$

where $\gamma$ is the surface tension of water that is positive. The water-accessible surface is the surface that is accessible to the centers of water molecules ${ }^{17}$. On the other hand, it is well known that $\mu$ of a small solute is substantially dependent on the excluded volume (EV) which is the volume enclosed by the water-accessible surface ${ }^{17}$. One might think that a protein is large enough to obey the scaling of Eq. (1), but this thought is inconsistent with cold denaturation caused by the weakening of the hydrophobicity at low temperatures. This inconsistency arises from the fact that as $T$ becomes lower, $\gamma$ increases: The scaling indicates that the hydrophobicity would become stronger. A protein possesses hydrophobic regions of widely varying length scales due to its complex polyatomic structure, which may distinguish it from a large spherical solute.

In the present study, we investigate the physical origin of the hydrophobicity by revisiting cold denaturation of a protein. Considering a completely solvophobic model protein ${ }^{18-23}$ immersed in water and three species of simple solvents for which the same solvent packing fraction is assumed, we analyze the temperature dependences of the changes in free energy, energy, and entropy of the solvent upon protein unfolding. The angle-dependent ${ }^{6,13-16}$ and radial-symmetric ${ }^{24-27}$ integral equation theories and the morphometric approach ${ }^{28-30}$ are employed in the analysis. The three species of simple solvents considered are (i) a solvent in which the particles interact through strongly attractive potential and the particle diameter is as small as that of water (simple solvent 1); (ii) a solvent in which the particles interact through strongly attractive potential but the particle diameter is about 1.9 times larger than that of water (simple solvent 2); and (iii) a hardsphere solvent whose particle diameter is the same as that of water. The microscopic mechanism of cold denaturation of a protein was studied in earlier works ${ }^{20,21,23}$, but the consideration of these simple solvents in the present work is expected to give a much larger amount of physically insightful information on the nature of the hydrophobicity. The morphometric approach allows us to decompose any of solvation thermodynamic quantities or its change upon protein unfolding into two terms which depend on the EV (term 1) and on the area and curvature of solvent-accessible surface (term 2), respectively. Effects due to the formation of ordered structure by the solvent molecules near the protein surface are included in term 2 . Term 1 , on the other hand, represents the contribution from the solvent molecules in the system with the proviso that the solvent molecules near the protein surface, which are influenced by the solute-solvent potential, are not included; See Subsection 2.5). For water and simple solvent 1, term 1 of the solvent-entropy change is further decomposed into two components representing the protein-solvent pair correlation and the protein-solvent-solvent triplet and higher-order correlation, respectively. The former is relevant to the total volume available to the translational displacement of solvent molecules. The latter is related to solvent crowding in the system. We note that the presence of a solvent molecule also generates an EV for the other solvent molecules, thus causing the solvent crowding. Such correlation among solvent molecules is not included in the protein-solvent pair correlation component.

The principal results can be summarized as follows. For water, the protein-solvent-solvent triplet and higher-order correlation component of term 1 of the negative entropy change upon protein unfolding becomes markedly smaller as $T$ decreases, which leads to cold denaturation of a protein induced by the weakening of the hydrophobicity. Water crowding in the system becomes more serious upon protein unfolding, but this effect becomes weaker as $T$ becomes lower. If the hydrophobicity originated from the structuring of water near a nonpolar solute as in the conventional view, it would be strengthened upon the temperature lowering. The rotationalentropy loss upon solute insertion is often emphasized for water, but only the water molecules near the solute contribute to it. Its effect is much less important than that of the translational entropy. Term 1 of the hydration free energy plays essential roles for a protein like for small nonpolar solutes such as methane. Among the three species of simple solvents, considerable weakening of the solvophobicity at low temperatures is observed only for simple solvent 1 . Even in the case of this solvent, however, cold denaturation cannot be reproduced. It would be reproducible if the attractive potential was substantially enhanced, but such enhancement gives rise to the appearance of the spinodal point beyond which the liquid state 
cannot exist as a single phase even in a metastable state. Water is unique in the sense that the hydrophobicity is powerful enough to form self-assembled structures at ambient temperature but substantially weakened when the temperature is lowered, leading to the collapse of the structures. We argue that the weakening of the solvophobicity at low temperatures is ascribed to enhanced local associations of solvent molecules in the bulk, causing more inhomogeneity followed by the formation of more void space: Due to the presence of more void space, the solvent can accommodate a solvophobic solute with less difficulty.

\section{Models and Theories}

\subsection{Change in system free energy upon protein unfolding}

We first consider a real protein immersed in aqueous solution. Diluted proteins are considered. The free-energy difference between the unfolded state and the native structure $\Delta G$ can be written as

$$
\Delta G(T)=\Delta E_{\mathrm{I}}+\Delta \mu(T)-T \Delta S_{\mathrm{C}}(T) .
$$

Here, $E_{\mathrm{I}}$ is the protein intramolecular energy and $S_{\mathrm{C}}(T)$ is the conformational entropy of the protein. $\Delta Z \equiv Z_{\mathrm{D}}-Z_{\mathrm{N}}$ denotes the change in a thermodynamic quantity upon the unfolding. The subscripts " $N$ " and " $D$ " represent the values for the native structure and for the unfolded (denatured) state, respectively. $\Delta G$, which is positive at ambient temperature, turns negative below the cold denaturation temperature. Under the isochoric condition, $\mu$ is expressed by

$$
\mu(T)=U_{V}(T)-T S_{V}(T) .
$$

The justification of considering the isochoric condition is discussed in Subsection 2.5. Equation (2) is then written as

$$
\Delta G(T)=\Delta E_{\mathrm{I}}+\Delta U_{V}(T)-T \Delta S_{V}(T)-T \Delta S_{\mathrm{C}}(T) .
$$

We decompose $\Delta U_{V}(T)$ into three terms as

$$
\Delta U_{V}(T)=\Delta U_{\mathrm{IN}}(T)+\Delta U_{\mathrm{RO}}(T)+\Delta U_{V, \mathrm{HS}}(T),
$$

where $\Delta U_{V, \mathrm{HS}}(T)$ is the change in the hydration energy calculated by replacing all the protein atoms by hard spheres (i.e., by modeling the protein as a set of fused hard spheres). $\Delta U_{\mathrm{IN}}(T)$ and $\Delta U_{\mathrm{RO}}(T)$ correspond to the changes in the protein-water interaction energy and in the water reorganization energy, respectively. These changes arise from the incorporation of the protein-water van der Waals and electrostatic interactions. Only the water molecules near the protein surface contribute to them. According to a recent experimental study $^{31}, \Delta E_{\mathrm{I}}+\Delta U_{\mathrm{IN}}(T)+\Delta U_{\mathrm{RO}}(T)+\Delta U_{V, \mathrm{HS}}(T)$ is negative at ambient temperature and it decreases further as $T$ becomes lower. For a solvent in which the solvent molecules interact through strongly attractive potential like water, $\Delta U_{V, \mathrm{HS}}(T)$ is negative and its absolute value becomes larger as the EV of a solute increases or $T$ decreases $^{6}$. Since the EV of the unfolded state is much larger than that of the native structure, $\Delta U_{V, \mathrm{HS}}(T)$ takes a large, negative value. Moreover, it decreases as $T$ becomes lower. The experimentally known behavior of $\Delta E_{\mathrm{I}}+\Delta U_{\mathrm{IN}}(T)+\Delta U_{\mathrm{RO}}(T)+\Delta U_{V, \mathrm{HS}}(T)$ mentioned above is reproducible by $U_{V . \mathrm{HS}}(T)$. We assume that $\Delta E_{\mathrm{I}}+\Delta U_{\mathrm{IN}}(T)+\Delta U_{\mathrm{RO}}(T)$ can be neglected for the following reason. When the protein unfolds, the protein intramolecular hydrogen bonds and van der Waals interactions are lost, leading to a positive value of $\Delta E_{\mathrm{I}}$. However, due to the proteinwater hydrogen bonds and van der Waals interactions gained, $\Delta U_{\mathrm{IN}}(T)$ takes a negative value. For the exposure of nonpolar groups $\Delta U_{\mathrm{RO}}(T)$ remains almost unchanged, while for that of polar and charged groups $\Delta U_{\mathrm{RO}}(T)$ becomes positive ${ }^{16}$. The three terms, $\Delta E_{\mathrm{I}}, \Delta U_{\mathrm{IN}}(T)$, and $\Delta U_{\mathrm{RO}}(T)$ are somewhat compensating ${ }^{32-34}$. As argued in our earlier work ${ }^{20}$, the neglect of $\Delta E_{\mathrm{I}}+\Delta U_{\mathrm{IN}}(T)+\Delta U_{\mathrm{RO}}(T)$ simply leads to a small shift of the cold denaturation temperature.

In general, $U_{V}$ and $\mu$ are largely dependent on the solutewater interaction potentials, while $S_{V}$ is considerably insensitive to them 35,36 . For example, using the three-dimensional reference interaction site model (3D-RISM) theory combined with all-atom potentials comprising Lennard-Jones (LJ) and Coulomb terms and the SPC/E water model, Imai et al. ${ }^{35} \mathrm{cal}-$ culated $S_{V}$ of the native structures of a total of eight peptides and proteins. Even when the protein-water electrostatic potentials, which are quite strong, are shut off and only the LJ potentials are retained, $S_{V}$ decreases merely by less than $5 \%$. In our earlier work ${ }^{36}, \mu, S_{V}$, and $U_{V}$ at $298.15 \mathrm{~K}$ were calculated for a hard-sphere solute with diameter of water molecule using the angle-dependent integral equation theory $6,13,14,16$ combined with the multipolar water model ${ }^{13,14}$. The calculated values are $\mu=5.95 k_{\mathrm{B}} T, S_{V}=-9.22 k_{\mathrm{B}}$, and $U_{V}=-3.27 k_{\mathrm{B}} T$. When the point charge $-0.5 e$ ( $e$ is the electronic charge) is embedded at its center, the calculated values are $\mu=-32.32 k_{\mathrm{B}} T$, $S_{V}=-10.11 k_{\mathrm{B}}$, and $U_{V}=-42.43 k_{\mathrm{B}} T: \mu$ and $U_{V}$ exhibit large decreases while $S_{V}$ remains roughly unchanged. Thus, $S_{V}$ can be approximated by $S_{\mathrm{V}, \mathrm{HS}}$ representing the hydration entropy calculated for the model protein, a set of fused hard spheres.

On the basis of the above discussion, Eq. (4) is approximately given by

$$
\Delta G(T) \simeq \Delta \mu_{\mathrm{HS}}(T)-T \Delta S_{\mathrm{C}}(T),
$$

where $\mu_{\mathrm{HS}}(T)=U_{V, \mathrm{HS}}(T)-T S_{V, \mathrm{HS}}(T)$ is the hydration free energy of the protein modeled as a set of fused hard spheres. Hereafter, the subscript "HS" is omitted (e.g., $\mu_{\mathrm{HS}}(T)$ is denoted simply by $\mu(T)$ ). We employ Eq. (6) by adopting a set of fused hard spheres which is completely solvophobic as 
the protein model for all the solvents considered. It should be noted that the details of the polyatomic structure, which is crucially important, are fully taken into account. $T \Delta S_{\mathrm{C}}(T)$ and $\Delta \mu(T)$ take large, positive values. $\Delta G(T)$ is positive at ambient temperature. $\Delta S_{\mathrm{C}}(T)$ is almost constant ${ }^{37}$ or a slightly increasing function of $T^{38}$. In either case, $-T \Delta S_{\mathrm{C}}(T)$ increases as $T$ becomes lower, shifting $\Delta G(T)$ in a more positive direction. Therefore, $\Delta \mu(T)$ must decrease to a sufficiently large extent for $\Delta G(T)$ to turn negative below the cold denaturation temperature. $\Delta \mu(T)$ is the key quantity for describing the stability of the native structure of a protein.

The heat-capacity change for the entire system upon protein denaturation has experimentally been shown to be positive ${ }^{9-11}$. It comprises the contributions from the hydration of nonpolar, polar, and charged groups and from the protein intramolecular energy. The contribution from the hydration of polar and charged groups is negative ${ }^{39,40}$ and that from the intramolecular energy is negligibly small ${ }^{41,42}$. Therefore, the contribution from the hydration of nonpolar groups, which is positive ${ }^{41,43}$, is dominant. This gives another justification of considering a completely hydrophobic model protein in the present study. Completely hydrophobic model proteins were considered in previous studies ${ }^{18-23}$ as well.

\subsection{Protein and solvent models}

The protein we consider is protein $\mathrm{G}$ with 56 residues [PDB code: 2GB1]. As explained above, the protein is modeled as a set of fused hard spheres. The $(x, y, z)$ coordinates of all the protein atoms (hydrogen, carbon, nitrogen, oxygen, etc.) in the backbone and side chains are used as part of the input data to account for the characteristics of each structure on the atomic level. The diameter of each atom is set at the $\sigma$-value of the LJ potential parameters of AMBER99. We assume that the unfolded state comprises a set of random coils*9. As in our earlier work ${ }^{20}, 32$ random-coil structures ${ }^{32}$ are employed as the unfolded state.

A water molecule is modeled as a hard sphere with diameter $d_{\mathrm{S}}=0.28 \mathrm{~nm}$ in which a point dipole and a point quadrupole of tetrahedral symmetry are embedded ${ }^{13,14}$. The influence of molecular polarizability of water is included by employing the self-consistent mean field (SCMF) theory ${ }^{13,14}$. At the SCMF level the many-body induced interactions are reduced to pairwise additive potentials involving an effective dipole moment. The number density of the bulk water $\rho_{\mathrm{S}}$ is taken to be that of real water along the saturation curve. The four temperatures, $258.15,263.15,273.15$, and $298.15 \mathrm{~K}$, are examined and

* It has recently been shown that yeast frataxin, which is denatured at 280 $\mathrm{K}$, possesses the properties of an unfolded protein at $272 \mathrm{~K}$ though a small amount of local, residual secondary structure is retained ${ }^{44}$. Our conclusions are not likely to be altered even when a completely unfolded state is considered as the cold-denatured one. the values of $\rho_{\mathrm{S}} d_{\mathrm{S}}^{3}$ at these temperatures are $0.7312,0.7325$, 0.7338 , and 0.7317 , respectively.

Particles of the simple solvents interact through

$$
\begin{array}{ll}
u_{\mathrm{SS}}=\infty & \text { for } \quad r<d_{\mathrm{S}} \\
u_{\mathrm{SS}}=-\varepsilon_{\mathrm{SS}}\left(\frac{d_{\mathrm{S}}}{r}\right)^{6} & \text { for } \quad r>d_{\mathrm{S}} .
\end{array}
$$

For "simple solvent 1 ", $d_{\mathrm{S}}$ and $\varepsilon_{\mathrm{SS}} /\left(k_{\mathrm{B}} T\right)$ are set at $0.28 \mathrm{~nm}$ and 1.6 at $298.15 \mathrm{~K}$, respectively. When $\varepsilon_{\mathrm{SS}}$ is set at zero, the solvent is formed by hard spheres and referred to as "hardsphere solvent". In simple solvent 1 and the hard-sphere solvent, $\rho_{\mathrm{S}} d_{\mathrm{S}}^{3}$ at each temperature is taken to be the same as that of water. It is physically insightful to look at the effect of the solvent diameter. To this end, we consider a simple solvent whose molecular diameter $d_{\mathrm{S}}^{\prime}$ is set at $0.53 \mathrm{~nm}$ that equals the $\sigma$-value of the LJ potential parameters for carbon tetrachloride $\left(\mathrm{CCl}_{4}\right)^{45}$. The number density $\rho_{\mathrm{S}}^{\prime}$ is evaluated so that $\rho_{\mathrm{S}}^{\prime} d_{\mathrm{S}}^{\prime 3}$ at each temperature becomes the same as $\rho_{\mathrm{S}} d_{\mathrm{S}}^{3}$ of water, and $\varepsilon_{\mathrm{SS}} /\left(k_{\mathrm{B}} T\right)$ is set at 1.6 at $298.15 \mathrm{~K}$ (the same as that for simple solvent 1$)$. We refer to this solvent as "simple solvent 2 ". We note that all the four solvents share the same packing fraction at each temperature.

\subsection{Integral equation theories}

The quantities we calculate are the hydration free-energy $\mu$, entropy $S_{V}$, and energy $U_{V}$ (they are referred to as the solvation free-energy, entropy, and energy, respectively, when all the solvents are simultaneously considered) for a protein with a prescribed structure. They are obtained through the integral equation theories and the morphometric approach ${ }^{28-30}$ described in the next subsection.

A hard-sphere solute of diameter $d_{\mathrm{U}}$ is immersed in solvent at infinite dilution. The solute-solvent correlation functions are calculated by the integral equation theory for the simple fluids ${ }^{24-27}$ and by its angle-dependent version for water ${ }^{6,13-16}$, and $\mu$ is obtained using the Morita-Hiroike formula ${ }^{46,47}$ or its extension to molecular liquids ${ }^{6,48}$. $S_{V}$ is evaluated through the numerical differentiation of $\mu$ with respect to $T^{6,48}$ as

$$
S_{V}=-\left(\frac{\partial \mu}{\partial T}\right)_{V}=-\frac{\mu(T+\delta T)-\mu(T-\delta T)}{2 \delta T}, \delta T=5 \mathrm{~K} .
$$

$U_{V}$ is obtained from $U_{V}=\mu+T S_{V}$. In the hard-sphere solvent, $U_{V}$ is zero and $\mu$ equals $-T S_{V}$. The solvation thermodynamic quantities are calculated for sufficiently many different values of $d_{\mathrm{U}}$ for determining the coefficients in the morphometric form (Eqs. (9) and (10)).

$S_{V}$ can be expressed as an expansion in terms of multiparticle correlation functions for a solute immersed in solvent ${ }^{49}$. On the basis of the expansion, we can decompose $S_{V}$ into the 
two components: the solute-solvent pair correlation component, $S_{V \text {,Pair }}$, and the solute-solvent-solvent triplet and higherorder correlation component, $S_{V \text {.Multi }}{ }^{50,51}$. $S_{V \text {.Pair }}$ is calculated using the solute-solvent pair correlation function and $S_{V, \text { Multi }}$ is obtained as $S_{V}-S_{V \text {.Pair }}$. Details of the decomposition were described in our previous papers ${ }^{50,51}$.

The radial-symmetric integral equation theory has been applied to a number of problems for simple fluids with successful results ${ }^{24-27}$. The reliability of the angle-dependent integral equation theory has also been verified in a number of studies. For example, the hydration free energies of small nonpolar solutes calculated by the theory combined with the multipolar water model are in perfect agreement with those from Monte Carlo simulations with the SPC/E and TIP4P water models ${ }^{6}$. The dielectric constant for bulk water, which is determined from the water-water orientational correlation functions, is in good agreement with the experimental data ${ }^{6}$. The theory is also capable of elucidating the hydrophilic hydration experimentally known ${ }^{16}$.

\subsection{Morphometric approach: decomposition of thermo- dynamic quantities of solvation and their changes upon protein folding}

In this approach, any of the solvation thermodynamic quantities is expressed using only four geometric measures of a solute with a fixed structure and corresponding coefficients ${ }^{28-30}$. The resultant morphometric form for the quantity $Z$ is given by

$$
Z=C_{1} V_{\mathrm{ex}}+C_{2} A+C_{3} X+C_{4} Y .
$$

Here, $V_{\mathrm{ex}}$ is the EV, $A$ is the ASA, and $X$ and $Y$ are the integrated mean and Gaussian curvatures of the water-accessible surface, respectively, and they form the four geometric measures. We calculate them for a protein with a prescribed structure by means of the extension ${ }^{29}$ of Connolly's algorithm ${ }^{52,53}$. In Eq. (9), the solute shape enters $Z$ only via the four geometric measures. Therefore, the four coefficients $\left(C_{1}-C_{4}\right)$ can be determined in simple geometries: They are determined from the values of $Z$ for hard-sphere solutes with various diameters (those in the range $0 \leq d_{\mathrm{U}} \leq 5 d_{\mathrm{S}}$; changing $5 d_{\mathrm{S}}$ to $10 d_{\mathrm{S}}$, for example, leads to no changes in the four coefficients determined). For the determination, we employ the angle-dependent (for water) or radial-symmetric (for the three species of simple solvents) integral equation theory. More details of the determination were described in our earlier publications ${ }^{21,48,51}$. Once the four coefficients are determined, $Z$ of a protein with any structure can be obtained by calculating only its four geometric measures.

For $Z$, we consider such quantities as $\mu, S_{V}$, and $U_{V} . Z$ can be decomposed into two terms. One of them consists of the second, third, and fourth terms in Eq. (9). This term, which is referred to as term 2, depends only on the area and curvatures of the solvent-accessible surface of the protein. It represents the contribution from the solvent molecules near the protein surface. The other is the first term in Eq. (9) which is referred to as term 1. The solvent molecules in the system, excluding those near the protein surface, contribute to term 1 depending on the EV of the protein. Terms 1 and 2 of $Z$ are denoted by $Z_{\text {Term1 }}$ and $Z_{\text {Term2 }}$, respectively.

We are concerned with $\Delta Z$ denoting the change in $Z$ upon protein unfolding. $Z$ of the unfolded state is calculated as its average value for the 32 random coils. With the present protein model, $\Delta \mu, \Delta U_{V}$, and $\Delta S_{V}$ correspond to the changes in free energy, energy, and entropy of the solvent upon the unfolding, respectively. It follows from Eq. (9) that $\Delta Z$ is expressed as:

$$
\Delta Z=C_{1} \Delta V_{\mathrm{ex}}+C_{2} \Delta A+C_{3} \Delta X+C_{4} \Delta Y
$$

As in the case of $Z$, we can discuss $\Delta Z$ by decomposing it into two terms, terms 1 and $2^{20,21}$. Term 2 , which consists of the second, third, and fourth terms in Eq. (10), is dependent only on the changes in the area and curvatures of the solventaccessible surface upon the unfolding. Term 1 is the first term in Eq. (10) which is influenced by the change in the EV. Terms 1 and 2 of $\Delta Z$ are denoted by $(\Delta Z)_{\text {Term } 1}$ and $(\Delta Z)_{\text {Term } 2 \text {, respec- }}$ tively.

In the case of water, any of the hydration thermodynamic quantities can be decomposed into translational and orientational components ${ }^{6}$. We emphasize that the translational component of $-T \Delta S_{V}$ possesses the EV term (term 1) while the orientational component does not ${ }^{48,50}$. The physical meaning of these components of $\Delta S_{V}$, for instance, is the following: Upon solute insertion, the translational and orientational freedoms of water molecules are reduced, causing losses of translational and rotational entropy of water, respectively. Only the water molecules near the solute undergo the orientational reduction, while the translational reduction reaches the water molecules in the bulk as well.

The usefulness of the morphometric approach has already been demonstrated. For example, the results from the threedimensional integral equation theory ${ }^{54,55}$ applied to the same model protein immersed in a simple solvent can be reproduced with sufficient accuracy by the morphometric approach applied to the same solvent ${ }^{29,48}$. By a hybrid of the angledependent integral equation theory combined with the multipolar water model and the morphometric approach, the experimentally measured changes in thermodynamic quantities upon apoPC folding are quantitatively reproduced ${ }^{48}$. Moreover, great progresses have been made in elucidating the microscopic mechanisms of pressure ${ }^{50,51}$, cold ${ }^{20,21}$, and heat ${ }^{56,57}$ denaturating of proteins, developing a physical picture for the rotation of $\mathrm{F}_{1}$-ATPase ${ }^{58}$, and discrimination of a native fold from misfolded decoys ${ }^{59-61}$ by our theoretical methods in which the morphometric approach is combined with 
the radial-symmetric integral equation theory or the angledependent version.

\subsection{Isochoric and isobaric conditions}

We consider the isochoric condition while the experiments are performed under the isobaric condition. This can be justified as follows. Under the isobaric condition, the changes in the solvent entropy and enthalpy upon protein unfolding, $\Delta S_{P}$ and $\Delta H$, are related to $\Delta S_{V}$ and $\Delta U_{V}$ through the thermodynamic relations ${ }^{15,62-64}$

$$
\begin{gathered}
\Delta S_{P} / k_{\mathrm{B}}=\Delta S_{V} / k_{\mathrm{B}}+\frac{\alpha^{*}}{\kappa_{T}^{*}} \frac{\Delta V_{P}}{d_{\mathrm{S}}^{3}}, \\
\Delta H /\left(k_{\mathrm{B}} T\right)=\Delta U_{V} /\left(k_{\mathrm{B}} T\right)+\frac{\alpha^{*}}{\kappa_{T}^{*}} \frac{\Delta V_{P}}{d_{\mathrm{S}}^{3}},
\end{gathered}
$$

where $V_{P}$ is the partial molar volume of the protein (i.e., change in the system volume upon protein insertion under the isobaric condition). The dimensionless parameters with the superscript "*”, which depend only on the properties of bulk solvent, are defined as

$$
\begin{aligned}
& \alpha^{*}=\alpha T, \\
& \kappa_{T}^{*}=\kappa_{T} k_{\mathrm{B}} T / d_{\mathrm{S}}^{3},
\end{aligned}
$$

where $\alpha$ is the isobaric thermal expansion coefficient and $\kappa_{T}$ is the isothermal compressibility.

We write $\Delta S_{V}$ and $\Delta U_{V}$ in Eq. (11) as

$$
\begin{aligned}
\Delta S_{V} & =\left(\Delta S_{V}\right)_{\mathrm{Term} 1}+\left(\Delta S_{V}\right)_{\mathrm{Term} 2} \\
& =C_{1, S_{V}} \Delta V_{\mathrm{ex}}+\left(\Delta S_{V}\right)_{\mathrm{Term} 2}, \\
\Delta U_{V} & =\left(\Delta U_{V}\right)_{\mathrm{Term} 1}+\left(\Delta U_{V}\right)_{\mathrm{Term} 2} \\
& =C_{1, U_{V}} \Delta V_{\mathrm{ex}}+\left(\Delta U_{V}\right)_{\mathrm{Term} 2 .}
\end{aligned}
$$

Here, $C_{1, S_{V}}$ or $C_{1, U_{V}}$ is the first coefficient in Eq. (10) applied to $S_{V}$ or $U_{V} . \Delta V_{P}$ can be expressed by

$$
\Delta V_{P}=\Delta V_{\mathrm{ex}}+\left(\Delta V_{P}\right)_{\mathrm{Term} 2} .
$$

Equation (11) is then given by

$$
\begin{aligned}
\Delta S_{P} / k_{\mathrm{B}} & =\left(C_{1, S_{V}} / k_{\mathrm{B}}+\frac{\alpha^{*}}{\kappa_{T}^{*}} \frac{1}{d_{\mathrm{S}}^{3}}\right) \Delta V_{\mathrm{ex}} \\
& +\left(\Delta S_{V} / k_{\mathrm{B}}\right)_{\mathrm{Term} 2}+\frac{\alpha^{*}}{\kappa_{T}^{*}} \frac{1}{d_{\mathrm{S}}^{3}}\left(\Delta V_{P}\right)_{\mathrm{Term} 2} \\
\Delta H /\left(k_{\mathrm{B}} T\right) & =\left(C_{1, U_{V}} /\left(k_{\mathrm{B}} T\right)+\frac{\alpha^{*}}{\kappa_{T}^{*}} \frac{1}{d_{\mathrm{S}}^{3}}\right) \Delta V_{\mathrm{ex}} \\
& +\left(\Delta U_{V} /\left(k_{\mathrm{B}} T\right)\right)_{\mathrm{Term} 2}+\frac{\alpha^{*}}{\kappa_{T}^{*}} \frac{1}{d_{\mathrm{S}}^{3}}\left(\Delta V_{P}\right)_{\mathrm{Term} 2}
\end{aligned}
$$

Since $\Delta V_{P}$ is experimentally known to be essentially zero in a wide range of $T^{31,65}$ :

$$
\begin{aligned}
\Delta V_{P} & \simeq 0, \\
\left(\Delta V_{P}\right)_{\mathrm{Term} 2} & \simeq-\Delta V_{\mathrm{ex}} .
\end{aligned}
$$

It follows that

$$
\begin{aligned}
\Delta S_{P} / k_{\mathrm{B}} & \simeq\left(C_{1, S_{V}} / k_{\mathrm{B}}\right) \Delta V_{\mathrm{ex}}+\left(\Delta S_{V} / k_{\mathrm{B}}\right)_{\mathrm{Term} 2}, \\
\Delta H /\left(k_{\mathrm{B}} T\right) & \simeq C_{1, U_{V}} /\left(k_{\mathrm{B}} T\right) \Delta V_{\mathrm{ex}}+\left(\Delta U_{V} /\left(k_{\mathrm{B}} T\right)\right)_{\mathrm{Term} 2} .
\end{aligned}
$$

It is apparent from Eq. (17) that

$$
\begin{aligned}
& \left(\Delta S_{P}\right)_{\mathrm{Term} 1} \simeq\left(\Delta S_{V}\right)_{\mathrm{Term} 1}, \\
& \left(\Delta S_{P}\right)_{\mathrm{Term} 2} \simeq\left(\Delta S_{V}\right)_{\mathrm{Term} 2}, \\
& (\Delta H)_{\mathrm{Term} 1} \simeq\left(\Delta U_{V}\right)_{\mathrm{Term} 1}, \\
& (\Delta H)_{\mathrm{Term} 2} \simeq\left(\Delta U_{V}\right)_{\mathrm{Term} 2} .
\end{aligned}
$$

Namely, $\Delta S_{P}, \Delta H$, and their terms 1 and 2 are approximately equal to $\Delta S_{V}, \Delta U_{V}$, and their terms 1 and 2, respectively. Due to the fact that $\left|\Delta V_{P}\right|$ is much smaller than $\left|\Delta V_{\mathrm{ex}}\right|$ as in Eq. (16), $\Delta S_{P}$ as well as $\Delta S_{V}$ possesses term 1 (i.e., the EV-dependent term). As shown in Sec. 3, term 1 plays essential roles even in $\mu$. This gives another evidence that the behavior of a sufficiently large solute expressed as Eq. (1), which is based on the assumption that term 1 of $\mu$ is negligibly small at ambient pressure $^{4,15}$, is not applicable to a protein.

\subsection{Estimation of conformation-entropy change upon protein unfolding at $298.15 \mathrm{~K}$}

The conformational-entropy change upon protein unfolding, $\Delta S_{\mathrm{C}}$, at $298.15 \mathrm{~K}$ can be estimated in the following manner. It is experimentally known for a number of proteins that $\Delta G$ in aqueous solution is approximately $\sim 50 \mathrm{~kJ} / \mathrm{mol}$ at 298.15 $\mathrm{K}^{66}$. We assume that protein $\mathrm{G}$ is no exception. Equation (6) applied to the case of $T=298.15 \mathrm{~K}$ then becomes

$$
\Delta \mu(298.15 \mathrm{~K})-298.15 \times \Delta S_{\mathrm{C}}=50 \mathrm{~kJ} / \mathrm{mol} \text {. }
$$

We can estimate $\Delta S_{\mathrm{C}}$ using Eq. (19) into which $\Delta \mu(298.15 \mathrm{~K})$ calculated for water through Eq. (10) is substituted. It is assumed that $\Delta S_{\mathrm{C}}$ takes the same value for all the solvents considered. Further, unless otherwise mentioned, $\Delta S_{\mathrm{C}}$ is treated as a constant that is independent of $T$. The value of $\Delta G$ for any of the three species of simple solvents is given by $\Delta \mu(298.15 \mathrm{~K})-298.15 \times \Delta S_{\mathrm{C}}$ where $\Delta \mu(298.15 \mathrm{~K})$ is calculated for the particular solvent.

\section{Results and discussion}

\subsection{Temperature dependence of protein solvophobicity}

The Ostwald coefficient $\exp (-\beta \mu)$ is a measure of the hydrophobicity, and we first look at the temperature dependence 
of $\beta \mu$. In Figures 1(a) and (b), $\beta \mu$ of the native structure of protein $\mathrm{G}$, which is modeled as a set of fused hard spheres, is plotted against $T$ for water and the three species of simple solvents. The decomposition of $\beta \mu$ into terms 1 and 2 is shown in Figure 1(c) (water), (d) (simple solvent 1), (e) (simple solvent 2), and (f) (hard-sphere solvent). We note that $\beta \mu$ of the unfolded state displays qualitatively the same characteristics (the data is not shown).

For water, $\beta \mu$ takes a large positive value $(\sim 630)$ at 298.15 $\mathrm{K}$ but decreases as $T$ becomes lower. It is observed in Figure 1(c) that this temperature dependence stems from that of term 1. Term 2, which is emphasized in the conventional view ${ }^{1}$, exhibits the opposite temperature $\mu$, term 1 , and term 2 for simple solvent 1 are qualitatively similar to those for water, though $\beta \mu$ and term 1 for simple solvent 1 are significantly larger.

The behavior for simple solvent 2 is substantially different from that for simple solvent 1 (compare Figures 1(d) and (e)) despite that these two simple solvents share the same attractive potential parameter. For the is essentially constant. The very minor change in $\beta \mu$ is merely due to the change in the solvent number density. Thus, the hydrophobicity can never be reproduced even in a qualitative sense when the strongly attractive potential is shut off. Sufficiently large $\beta \mu$ at ambient temperature and the appreciable reduction in $\beta \mu$ upon the lowering of $T$ is attributed to the interplay of the exceptionally sspectively.

As observed in Figure 2(a) for water, $\Delta \mu$ decreases as $T$ becomes lower: It decreases by $\sim 115 \mathrm{~kJ} / \mathrm{mol}$ upon the lowering of $T$ from $298.15 \mathrm{~K}$ to $258.15 \mathrm{~K}$. Both of $\Delta U_{V}$ (Figure 2(b)) and $\Delta S_{V}$ (Figure 2(c)) are negative and they decrease further as $T$ becomes lower. It has experimentally been shown that the changes in enthalpy and entropy for the entire system upon protein unfolding are negative at ambient temperature and decrease further as $T$ becomes lower ${ }^{31}$. The temperature dependences of $\Delta U_{V}$ and $\Delta S_{V}$ are consistent with this experimental result. It is observed in Figure 3(a) for simple solvent 1 that $\Delta S_{V}$ and $\Delta U_{V}$, which are both negative, decrease further as $T$ becomes lower. $\Delta \mu$ decreases by $\sim 110 \mathrm{~kJ} / \mathrm{mol}$ upon the lowering of $T$ from $298.15 \mathrm{~K}$ to $258.15 \mathrm{~K}$. These characteristics are qualitatively similar to those observed for water.

We now discuss the temperature dependences of terms 1 and 2 of $\Delta \mu, \Delta U_{V}$, and $-T \Delta S_{V}$ or $\Delta S_{V}$. It is found that the two terms exhibit similar dependences on $T$ for water and simple solvent 1 . The temperature dependence of $-T \Delta S_{V}$ or that of $\Delta U_{V}$ is governed by that of term 2. Term 2 of $-T \Delta S_{V}$ increases while that of $\Delta U_{V}$ decreases as $T$ becomes lower. The increase and decrease are somewhat compensating but the increase is more or less larger: Term 2 of $\Delta \mu$ does not decrease as $T$ becomes lower. Thus, cold denaturation cannot be induced by term 2 of $\Delta \mu$. A closer look at Figures 2 and 3 allows us to appreciate the following difference between water and simple solvent 1 . For water, term 2 of $\Delta U_{V}$ or $\Delta S_{V}$ decreases more sharply with decreasing $T$ : the sign of term 2 of $\Delta S_{V}$ turns negative at $\sim 265 \mathrm{~K}$ and that of $\Delta U_{V}$ is also likely to turn negative at a temperature lower than $258.15 \mathrm{~K}$. This behavior, which is not shared by simple solvent 1 , is attributable to the formation of highly ordered structure of water near the protein surface due to the enhancement of hydrogen bonding or increase in the number of hydrogen bonds. Compared with the native structure, the unfolded state possesses much larger ASA and more water molecules participating in the orderedstructure formation, leading to larger entropic loss and energy decrease upon the unfolding at lower temperatures. However, this entropic loss and energy decrease are almost cancelled out and term 2 of $\Delta \mu$ remains almost unchanged.

As argued in our earlier publications ${ }^{20,21}$, the decrease in $\Delta \mu$ by $\sim 115 \mathrm{~kJ} / \mathrm{mol}$ mentioned above for water induces cold denaturation of our model protein at $\sim 259 \mathrm{~K}$. This behavior originates from the temperature dependence of term 1. The decrease in term 1 of $-T \Delta S_{V}$, which surpasses the increase in term 1 of $\Delta U_{V}$, is the cause of cold denaturation. Here we decompose term 1 of $-T \Delta S_{V}$ into the two components, $-T \Delta S_{\mathrm{V}, \text { Pair }}$ and $-T \Delta S_{\mathrm{V}, \text { Multi }}$, representing the proteinwater pair correlation and the protein-water-water triplet and higher-order correlation, respectively. A similar decomposition is performed for simple solvent 1 . The results for water and simple solvent 1 are compared in Figures 4(a) and (b). We note that $C_{1}$ of $S_{V \text {,Pair }}$ is $\rho_{\mathrm{S}}{ }^{50,51}$ (only $S_{V \text {,Pair }}$ is incorporated in the Asakura-Oosawa theory ${ }^{67,68}$ ). For both of the two solvents, $-T \Delta S_{\mathrm{V}, \text { Multi }}$ is substantially larger than $-T \Delta S_{\mathrm{V}, \text { Pair }}$. The temperature dependence of term 1 of $-T \Delta S_{V}$ arises from that of $-T \Delta S_{\mathrm{V}, \text { Multi }}$. As a significant difference, for water, $-T \Delta S_{\mathrm{V}, \text { Multi }}$ decreases more sharply as $T$ becomes lower. The weakening of the hydrophobicity followed by cold denaturation in water is induced by the EV-dependent term of the hydration entropy at the protein-solvent-solvent triplet and higher-order correlation level.

The EV term (term 1) of $-T \Delta S_{V}$ is nothing but its translational component (the orientational component possesses no EV term ${ }^{48,50}$; see Subsection 2.4). It should be emphasized that the presence of a water molecule generates an EV for the other water molecules, thus causing water crowding. The EV of the native structure is much smaller than that of the unfolded state ${ }^{48,69}$. Upon protein folding, the water crowding in the system is substantially reduced, leading to a large gain in the translational entropy of water ${ }^{48,69}$. This gain is a consequence of the protein-solvent-solvent triplet and higher-order correlation and the driving force of protein folding. However, this force becomes much less powerful at low temperatures, giving rise to cold denaturation.

As mentioned above, it is experimentally known that the heat-capacity change for the entire system upon protein denaturation is positive ${ }^{9-11}$, implying the dominant contribution 
from the hydrophobic hydration. Since $\Delta U_{V}$ and $\Delta S_{V}$ for water is an increasing function of $T$ as shown in Figures 2(b) and (c), $\Delta C_{V}$ is positive, which is consistent with the experimental result. As far as the temperature dependence of $\Delta U_{V}$ or $\Delta S_{V}$ is concerned, it is governed by term 2. This indicates that $\Delta C_{V}$ is determined primarily by the contribution from hydrogen bonds of the water molecules near the protein surface. Our result never conflicts with the empirical picture that the change in the heat capacity upon protein denaturation can be scaled by that in the $\mathrm{ASA}^{70}$. It is worthwhile to note that $\Delta C_{V}$ is much larger for water than for simple solvent 1 probably due to the hydrogen-bonding property (see Figures 2 and 3).

\subsection{Temperature dependences of changes in thermody- namic quantities of simple solvent 2 and hard-sphere solvent upon protein unfolding}

The temperature dependences of the changes in thermodynamic quantities of simple solvent 2 and hard-sphere solvent upon protein unfolding are shown in Figures 5 (simple solvent 2) and 6 (hard-sphere solvent). For the hard-sphere solvent, $\Delta U_{V}=0$ and $\Delta \mu=-T \Delta S_{V}$.

By comparing Figures 3 and 5, we notice that the increase of the solvent diameter by 1.9 times leads to a drastic change in the behavior of thermodynamic quantities: The absolute values of $\Delta \mu, \Delta U_{V}, \Delta S_{V}$, and $-T \Delta S_{V}$ become substantially smaller and they exhibit much weaker temperature dependences. Qualitatively the same characteristics are observed for their terms 1 and 2. As for the hard-sphere solvent (Figure 6), $\Delta S_{V}$ is essentially independent of $T$ and $\Delta \mu=-T \Delta S_{V}$ decreases simply in proportion to $T$ as $T$ becomes lower. The absolute value of $\Delta S_{V}$ for the hard-sphere solvent is much larger than for simple solvent 2 , which should be due to the smaller solvent diameter. However, the hard-sphere solvent and simple solvent 2 share the behavior that $\Delta S_{V}$ remains almost constant against a temperature change. The experimentally known result ${ }^{31}$, "the changes in enthalpy and entropy for the entire system upon protein unfolding exhibit considerable decrease with lowering $T^{\prime \prime}$, can never be explained.

In summary, with respect to the temperature dependences of changes in thermodynamic quantities of solvation upon protein unfolding, water and simple solvent 1 exhibit qualitatively similar behavior. The increase in the solvent diameter by 1.9 times or removal of the solvent-solvent attractive interaction potential in simple solvent 1 leads to drastically different behavior. The interplay of the exceptionally small molecular size and strongly attractive interaction of the solvent is important in reproducing the behavior of water. However, there are certainly some differences in details of the behavior between water and simple solvent 1 , which is further discussed in a later subsection.

\subsection{Change in thermodynamic quantities of entire sys- tem upon protein unfolding at ambient temperature}

Table 1 shows $\Delta \mu,-T \Delta S_{\mathrm{C}}$, and $\Delta G$ upon protein unfolding at $298.15 \mathrm{~K}$ calculated for water and the three species of simple solvents in accordance with the procedure described in Subsection 2.6. According to the argument described in ref 48, $-T \Delta S_{\mathrm{C}}$ of protein $\mathrm{G}$ should be in the range, $-541 \mathrm{~kJ} / \mathrm{mol}$ $<-T \Delta S_{\mathrm{C}}<-173 \mathrm{~kJ} / \mathrm{mol}$. The value of $-T \Delta S_{\mathrm{C}}$ in Table 1 is certainly in this range, proving the validity of the value. For simple solvent 1 or the hard-sphere solvent $\Delta G$ takes a larger positive value than for water. For simple solvent $2, \Delta \mu$ is much smaller than for any of the other solvents, leading to the negative sign of $\Delta G$. This means that the unfolded state is more stable than the native structure even at ambient temperature. A solvent with too large a value of the molecular diameter is not capable of driving a protein to fold. Simple solvent 1 and the hard-sphere solvent possess this capability, but cold denaturation, which can be caused by the weakening of the solvophobicity at low temperatures, is not reproducible for the model protein immersed in these solvents as discussed below.

\subsection{Possibility of cold denaturation of a protein in water, simple solvent 1, and hard-sphere solvent}

Since $\Delta G=\Delta \mu-T \Delta S_{\mathrm{C}}$, the cold denaturation temperature is the temperature at which $\Delta \mu=T \Delta S_{\mathrm{C}}$. We assume that $\Delta S_{\mathrm{C}}$ is independent of $T^{37}$. The temperature dependence of $\Delta \mu$ for water and that of $T \Delta S_{\mathrm{C}}$ are illustrated in Figure 7(a). The two lines intersect at $\sim 259 \mathrm{~K}$ being the denaturation temperature. This temperature is quite consistent with the experimentally observed one $(250 \mathrm{~K} \sim 260 \mathrm{~K})^{9-11}$. Even if $\Delta S_{\mathrm{C}}$ is treated as an increasing function of $T, T \Delta S_{\mathrm{C}}$ decreases a little more rapidly as $T$ becomes lower, leading to only a small shift of the denaturation temperature in a lower direction.

Figure 7(b) shows the temperature dependence of $\Delta \mu$ for simple solvent 1 and that of $T \Delta S_{\mathrm{C}}$. By extrapolating the data of $\Delta \mu$ to the temperatures lower than $258.15 \mathrm{~K}$, we estimate that the two lines intersect at $\sim 160 \mathrm{~K}$. This temperature is unrealistically low. Moreover, using the radial-symmetric integral equation theory, we find that the spinodal point at which the isothermal compressibility diverges is encountered at $\sim$ $222 \mathrm{~K}$ when $T$ is progressively lowered. This means that simple solvent 1 cannot exist as a single liquid phase even in the metastable state below $\sim 222 \mathrm{~K}$. It is concluded that cold denaturation is not reproducible in this solvent.

In the hard-sphere solvent, $\Delta \mu$ is equal to $-T \Delta S_{V}$ and $\Delta G$ is expressed as

$$
\Delta G(T)=-T\left(\Delta S_{V}+\Delta S_{\mathrm{C}}\right) .
$$

$\Delta S_{V} / k_{\mathrm{B}}$ and $\Delta S_{\mathrm{C}} / k_{\mathrm{B}}$ at ambient temperature are -284.2 and 111.2, respectively, as given in Table 1 . We note that $\Delta S_{V}$ 
remains almost constant against the temperature lowering (see Figure 6(a)). Hence, cold denaturation cannot occur when $\Delta S_{\mathrm{C}}$ is assumed to be independent of $T$. Even if we consider that $\Delta S_{\mathrm{C}}$ decreases as $T$ becomes lower, $\Delta G$ can never become 0 because $\left|\Delta S_{V} / k_{\mathrm{B}}\right|>\Delta S_{\mathrm{C}} / k_{\mathrm{B}}$ at ambient temperature and $\Delta S_{V}$ remains almost constant. Thus, cold denaturation is not likely to occur in the hard-sphere solvent.

\subsection{Further comparison between water and simple sol- vent 1}

The solvophobicity is considerably weakened at low temperatures for simple solvent 1 as well as for water. However, we find the following differences between these solvents. As discussed above, for water at low temperatures, highly ordered structure is formed near the protein surface due to the enhancement of hydrogen bonding or increase in the number of hydrogen bonds. For simple solvent 1, on the other hand, we find no appreciable sign of such solvent structuring near the protein surface.

Cold denaturation of a protein is reproducible in water but not in simple solvent 1 . This is partly because the temperature dependence of the EV-dependent term of the hydration entropy at the protein-solvent-solvent triplet and higher-order correlation level is stronger for water. Another reason is in $\Delta \mu$ of simple solvent 1 at ambient temperature which is significantly larger than that of water (see Table 1). The difference between water and simple solvent 1 in $\Delta \mu$ can be explained as follows. As shown in Figures 2(d) and 3(d), the two solvents share essentially the same value of $-T \Delta S_{V}$. On the other hand, $\Delta U_{V}$ is substantially more negative for water than for simple solvent 1 (see Figures 2(b) and 3(b)). For a solvent whose particles interact through attractive interaction, the solute insertion causes the internal energy to decrease. Since the direct solvent-solute interaction does not contribute energetically for our solute model, the energy decrease is due to the structural changes induced in the solvent. $U_{V}=0$ for the hard-sphere solvent but $U_{V}$ take a large, negative value for simple solvent 1 or water. $U_{V}$ of the unfolded state of a protein $\left(U_{V, \mathrm{D}}\right)$ is more negative than that of its native structure $\left(U_{V, \mathrm{~N}}\right): \Delta U_{V}=U_{V, \mathrm{D}}-U_{V, \mathrm{~N}}<0$. Further, $U_{V, \mathrm{D}}, U_{V, \mathrm{~N}}$, and $\Delta U_{V}$ for water are substantially more negative than $U_{V, \mathrm{D}}$, $U_{V, N}$, and $\Delta U_{V}$ for simple solvent 1 , respectively, due to the considerably stronger attractive interaction. Thus, the difference between the two solvents in $\Delta \mu$ arises from that in the strength of the solvent-solvent attractive interaction giving rise to the internal-energy decrease upon solute insertion. (When the temperature is lowered from $298.15 \mathrm{~K}$ to $258.15 \mathrm{~K}$, the decrease in $\Delta U_{V}$ for water is larger than that for simple solvent 1 as expected, while the increase in $-T \Delta S_{V}$ for water is also larger than for simple solvent 1 by almost the same magnitude: Due to the cancellation, the two solvent share almost the same decrease in $\Delta \mu$. The larger increase in $-T \Delta S_{V}$ for water originates from the enhanced hydrogen bonding near the protein surface occurring in the very low temperature range as discussed above.)

We find that $\Delta \mu$ of simple solvent 1 at ambient temperature becomes closer to that of water when the solvent-solvent attractive potential is enhanced by increasing $\varepsilon_{\mathrm{SS}}\left(\rho_{\mathrm{S}} d_{\mathrm{S}}^{3}\right.$ is fixed at 0.7317 ). However, the radial-symmetric integral equation theory looses its solution before $\beta \mu$ for simple solvent 1 becomes sufficiently close to that for water when $\varepsilon_{S S}$ is progressively increased: The divergence of the isothermal compressibility of the bulk solvent is encountered. Thus, there are certainly some aspects in the water behavior which cannot be reproduced by a simple model solvent with no hydrogen bonds.

As pointed out above, the interplay of the exceptionally small molecular size and strongly attractive interaction of the solvent is essential in mimicking the water behavior. At the same time, hydrogen bonds are necessitated for the complete elucidation of the hydrophobicity. Water is unique in the sense that its hydrophobicity is powerful enough to form selfassembled structures at ambient temperature but substantially weakened when the temperature is lowered, leading to the collapse of the structures. This feature cannot be reproduced even by simple solvent 1 that is far closer to water in solvation thermodynamics than simple solvent 2 and the hard-sphere solvent.

\subsection{Physical origin of weakening of solvophobicity at low temperatures for water and simple solvent 1}

It is clear that a sufficiently strong attractive interaction between solvent particles is required for reproducing the weakening of the solvophobicity at low temperatures. Consider first the bulk solvent. As the temperature becomes lower, the effect of the attractive interaction becomes stronger, reducing the number of accessible translational configurations of solvent particles due to the constraints caused by the effect. For water, the contact value of the solvent-solvent pair correlation function $g\left(d_{\mathrm{S}}\right)$ is 18.2 at $298.15 \mathrm{~K}$ but it increases to 22.1 at $258.15 \mathrm{~K}$. For the simple solvent 1 , it is 6.11 at $298.15 \mathrm{~K}$ but it increases to 6.56 at $258.15 \mathrm{~K}$. This type of increase represents that associations of solvent molecules are locally enhanced at low temperatures. Since the solvent number densities at $298 \mathrm{~K}$ and at $258 \mathrm{~K}$ share almost the same value (i.e., the system volume remains almost unchanged), the enhanced associations accompany an increase in the inhomogeneity of the solvent, producing more void space. Due to the presence of more void space, the decrease in the number of accessible translational configurations of solvent molecules upon the solute insertion becomes smaller, the degree of the enhancement of solvent crowding reduces, and the entropic loss becomes less serious. Thus, the solvent can accommodate a solvopho- 
bic solute with less difficulty (i.e., the solvophobicity is weakened) at low temperatures. Judging from the values of $g\left(d_{\mathrm{S}}\right)$ given above, this temperature effect is much larger for water than for simple solvent 1 , because the solvent-solvent attractive interaction in simple solvent 1 is considerably weaker.

Matubayasi and Nakahara investigated the effect of the dipole moment of water molecules on $\mu$ of a nonpolar solute using a computer simulation ${ }^{71}$. The simulation was performed under the isochoric condition. They found that $\mu$ decreases as the dipole moment becomes larger. It was then argued that the enhanced hydrogen bonding arising from the increased dipole moment gives rise to more void space, leading to a reduction in $\mu$. This interpretation is closely related and similar to ours described above.

The water density decreases as the temperature becomes lower from $277 \mathrm{~K}$. However, this decrease is very minor and cannot cause cold denaturation of a protein by itself. To demonstrate this, we calculate $\Delta \mu$ for water at $258.15 \mathrm{~K}$ with two number densities: the values pertinent to 258.15 $\mathrm{K}\left(\rho_{\mathrm{S}} d_{\mathrm{S}}^{3}=0.7312\right)$ and $298.15 \mathrm{~K}\left(\rho_{\mathrm{S}} d_{\mathrm{S}}^{3}=0.7317\right)$, respectively. The result is given as Table 2: $\Delta \mu$ and its terms 1 and 2 with $\rho_{\mathrm{S}} d_{\mathrm{S}}^{3}=0.7317$ are almost indistinguishable from those with $\rho_{\mathrm{S}} d_{\mathrm{S}}^{3}=0.7312$. Thus, the weakening of the hydrophobicity at low temperatures and cold denaturation of a protein are ascribed solely to the solvent-solvent attractive interaction coupled with the temperature lowering. Graziano succeeded in reproducing cold denaturation of a protein using the classical scaled particle theory (SPT) ${ }^{23}$. The SPT, in which the solvent is always formed by hard spheres, is capable of explicitly incorporating neither the solvent-solvent attractive interaction nor the temperature effect. In his work, however, the hardsphere diameter for the solvent is adjusted so that the experimental value of the isothermal compressibility of water can be fitted with respect to the SPT relationship. The resulting diameter is referred to as "effective diameter". The effective diameter decreases as the temperature becomes lower below 20 ${ }^{\circ} \mathrm{C}$. The decrease in the total packing fraction of the solvent at low temperatures, which leads to the reduction in the work of cavity creation and the occurrence of cold denaturation, arises mostly from the smaller effective diameter. The decrease in the solvent density itself has only very minor effects. In our view, the effects of the solvent-solvent attractive interaction coupled with the temperature lowering are implicitly incorporated in the treatment of Graziano through the adjustment of the diameter. In this sense, our argument is never inconsistent with that of Graziano.

\section{Conclusions}

We have investigated the physical origin of the hydrophobicity by revisiting cold denaturation of a protein. There are a number of phenomena manifesting that the hydrophobicity is weakened at low temperatures, and cold denaturation is a typical example. In order to explore the feature of water, three species of simple solvents as well as water are considered in the investigation. Considering a completely solvophobic model protein, we analyze the temperature dependences of the solvation free energy multiplied by $\beta=1 /\left(k_{\mathrm{B}} T\right)$ $(\beta \mu)$ of a folded protein, a measure of the solvophobicity. Those of the changes in free energy $\Delta \mu$, energy $\Delta U_{V}$, and entropy $\Delta S_{V}$ (or $-T \Delta S_{V}$ ) of the solvent upon protein unfolding are also analyzed. The angle-dependent ${ }^{6,13-16}$ and radialsymmetric $^{24-27}$ integral equation theories and the morphometric approach ${ }^{28-30}$ are employed in the analysis. The three species of simple solvents considered are as follows: simple solvent 1 in which the particles interact through strongly attractive potential and the particle diameter is as small as that of water; simple solvent 2 in which the particles interact through strongly attractive potential but the particle diameter is about 1.9 times larger than that of water; and a hardsphere solvent whose particle diameter is the same as that of water. The four solvents share the same packing fraction at each temperature. The changes in thermodynamic quantities of the solvent upon protein unfolding are decomposed into two terms, term 1 which is scaled by the excluded volume (EV) and term 2 depending on the area and curvature of solventaccessible surface, using the morphometric approach. The solvent molecules near the protein surface and those in the system (excluding those near the protein surface) contribute to term 2 and to term 1, respectively. Term 2 includes the effects due to the formation of ordered structure by the solvent molecules near the protein surface. For water and simple solvent 1, term 1 of $-T \Delta S_{V}$ is further decomposed into the proteinsolvent pair correlation component and the protein-solventsolvent triplet and higher-order correlation component. The former is relevant to the total volume available to the translational displacement of solvent molecules. The latter is related to the solvent crowding in the system which is caused by the physical factor that the presence of a solvent molecule also generates an EV for the other solvent molecules. Such correlation among solvent molecules is not included in the proteinsolvent pair correlation component.

The following characteristics of water can qualitatively be reproduced by simple solvent 1: (i) $\beta \mu$ decreases (i.e., the hydrophobicity is weakened) considerably as $T$ becomes lower; (ii) $\Delta \mu$ reduces considerably with lowering $T$ due to the decrease in its term 1 which is ascribed to the reduction in term 1 of $-T \Delta S_{V}$; and (iii) the reduction in term 1 of $-T \Delta S_{V}$ originates from that in its protein-solvent-solvent triplet and 
higher-order correlation component. For water, term 1 of $\mu$ plays essential roles for a protein just as it does for small nonpolar solutes such as methane. This is an important point suggesting that the scaling behavior of Eq. (1) does not hold for a protein. The results for simple solvent 2 and the hardsphere solvent are substantially different from those for water and simple solvent 1: The temperature dependences of $\beta \mu$, $\Delta \mu, \Delta U_{V}$, and $\Delta S_{V}$ (or $-T \Delta S_{V}$ ) are much weaker. It is suggested that the water characteristics are ascribed to the interplay of the two factors, the exceptionally small molecular size and strongly attractive interaction. If one of the two factors is absent, the characteristics of water are lost. Even between water and simple solvent 1 , the following differences are appreciated in the behavior: (i) The temperature dependences of term 1 of $-T \Delta S_{V}$ and its protein-solvent-solvent triplet and higher-order correlation component are significantly stronger for water; (ii) though the two solvents share the result that the temperature dependences of $\Delta U_{V}$ and $\Delta S_{V}$ are governed by their term 2 but they are compensating, the dependences for water are much stronger; and (iii) there is a clear sign of water structuring arising from the enhanced hydrogen bonding near the protein surface at low temperatures whereas such a sign is not found for simple solvent 1.

Not only water but also simple solvent 1 and the hardsphere solvent are capable of driving a protein to fold while simple solvent 2 is not. Only in water, the solvophobicity becomes weak enough to give rise to protein unfolding at low temperatures. Cold denaturation is not reproducible even in simple solvent 1 due to the insufficient weakening of the solvophobicity despite that water and this solvent share some qualitatively similar characteristics as described above. It would be reproducible if the attractive potential was substantially enhanced, but such enhancement causes the appearance of the metastability limit for a single liquid phase. In this sense, the hydrogen bonds are related to the exhibition of the hydrophobicity. However, this never implies the importance of the orientational component of the hydration entropy. It is relevant only to the water molecules near the protein surface and much smaller than the translational component. Water is unique in the sense that the hydrophobicity is powerful enough to form a variety of self-assembled structures of solute molecules at ambient temperature but substantially weakened when the temperature is lowered, leading to the collapse of the structures. This uniqueness is ascribed to the reduction in the EV term of the hydration entropy at the solute-solvent-solvent triplet and higher-order correlation level. It should be emphasized that the presence of a water molecule generates an EV for the other water molecules, thus causing water crowding. Upon a self-assembly process, the water crowding in the system is substantially reduced with the result of a large gain in the translational entropy of water. However, this force driving the process becomes much less powerful at low temperatures, giving rise to the collapse of the self-assembled structures. In our view, the weakening of the hydrophobicity at low temperatures leading to cold denaturation of a protein is attributable to enhanced local associations of water molecules in the bulk, giving rise to more inhomogeneity followed by the formation of more void space: Due to the presence of more void space, water can accommodate a hydrophobic solute with less difficulty. We believe that the same physical origin is shared by the following phenomena at low temperatures: The solubility of methane increases, the critical micelle concentration becomes higher, the average size of micelles for nonionic amphiphilic molecules becomes smaller, most of the native structures of proteins unfold, and protein aggregation is dissociated.

As described in Introduction, the behavior of the hydration free energy $\mu$ (the solvent is water) of a sufficiently large solute is quite different from that of a small solute ${ }^{4}$ : While $\mu$ of the former is scaled by the water-accessible surface area, $\mu$ of the latter is largely dependent on the excluded volume. We note that the four coefficients in the morphometric form for $\mu$ are determined by the fitting to the hydration free energies of hard-sphere solutes. The resulting values of the four coefficients can largely be influenced by the solute sizes chosen in the fitting. $C_{1}$ and $C_{2}$ could be approximated by $P$ (the pressure) and $\gamma$ (the surface tension), respectively, when only sufficiently large hard-sphere solutes are employed in the fitting. On the other hand, the coefficients adopted in our studies are determined from small hard-sphere solutes (see Sec. 2.4). In this case, $C_{1}$ and $C_{2}$ are substantially different from $P$ and $\gamma$, respectively. Our proposition is that the coefficients determined using sufficiently small hard-sphere solutes should be employed for proteins in order to reproduce the experimentally observed behavior of cold denaturation. Namely, the first term of the morphometric form for $\mu$ is not the pressurevolume work. We have tackled a number of problems related to solvation thermodynamics of a variety of solutes. On the basis of the results obtained, we now believe that the largesolute limit is inapplicable to proteins as well as to small solutes like methane (this was already stated in our paper, Ref. 20) when the solvent is water. At the large-solute limit, with $P=1 \mathrm{~atm}$, the first term in the morphometric form can be neglected. With the absence of the first term, however, many of the important problems (e.g., the very large entropic gain upon folding of apoplastocyanin, cold and pressure denaturating of a protein, and thermal stability of a protein) cannot be elucidated. In summary, the first term in the morphometric form for $\mu$ is quite large even for water with $P=1 \mathrm{~atm}$; and the second and third terms of the morphometric form also make significantly large contributions to $\mu$. On the other hand, for the hard-sphere solvent, when the four coefficients are determined using hard-sphere solutes with diameter $d_{\mathrm{U}}$ ranging from $d_{\mathrm{U} 1}$ to $d_{\mathrm{U} 2}$, the resulting values are almost completely independent of $d_{\mathrm{U} 1}$ and $d_{\mathrm{U} 2}$ chosen. Since the large-solute 
limit seems to be applicable to a protein immersed in the hardsphere solvent, the morphometric form in Refs. 29 and 72 was written for this limit. We remark that the large-solute limit is not applicable to a protein immersed in simple solvent 1 as in the case of water.

It is of great interest and importance to further examine whether or not the proposition given in the present study is universally applicable to the behavior of diverse self-assembly processes. Work in this direction is in progress.

\section{Acknowledgments}

The computer program for the morphometric approach was developed with Roland Roth and Yuichi Harano. This work was supported by Grants-in-Aid for Scientific Research on Innovative Areas (No. 20118004) from the Ministry of Education, Culture, Sports, Science and Technology of Japan and by the Grand Challenges in Next-Generation Integrated Nanoscience, MEXT, Japan.

\section{References}

1 W. Kauzmann, Adv. Protein Chem., 1959, 14, 1-63.

2 K. A. Dill, Biochemistry, 1990, 29, 7133-7155.

3 B. Widom, P. Bhimalapuram, and K. Koga, Phys. Chem. Chem. Phys., 2003, 5, 3085-3093.

4 D. Chandler, Nature, 2005, 437, 640-647.

5 H. S. Ashbaugh and L. R. Pratt, Rev. Mod. Phys., 2006, 78, 159-179.

6 M. Kinoshita, J. Chem. Phys., 2008 128, 024507(1-14).

7 G. Graziano, Chem. Phys. Lett., 2012, 533, 95-99.

8 D. Myres, Surfaces, Interfaces, and Colloids: Principles and Applications, Wiley-VCH, Berlin, 1999.

9 A. Pastore, S. R. Martin, A. Politou, K. C. Kondapalli, T. Stemmler, and P. A. Temussi, J. Am. Chem. Soc., 2007, 129, 5374-5375.

10 P. L. Privalov, Y. V. Griko, S. Yu. Venyaminov, and V. P. KutyShenko, J. Mol. Biol., 1986, 190, 487-498.

11 P. L. Privalov, Crit. Rev. Biochem. Mol. Biol., 1990, 25, 281-306.

12 R. Mishra, and R. Winter, Angew. Chem. Int. Ed., 2008, 47, 6518-6521.

13 P. G. Kusalik and G. N. Patey, J. Chem. Phys., 1988, 88, 7715-7738.

14 P. G. Kusalik and G. N. Patey, Mol. Phys., 1988, 65, $1105-$ 1119.

15 N. M. Cann and G. N. Patey, J. Chem. Phys., 1997, 106, 8165-8195.

16 M. Kinoshita and T. Yoshidome, J. Chem. Phys., 2009, 130, 144705(1-11).
17 B. Lee and F. M. Richards, J. Mol. Biol., 1971, 55, 379400.

18 S. V. Buldyrev, P. Kumar, P. G. Debenedetti, P. J. Rossky, and H. E. Stanley, Proc. Natl. Acad. Sci. U.S.A., 2007, 104, 20177-20182.

19 C. L. Dias, T. Ala-Nissila, M. Karttunen, I. Vattulainen, and M. Grant, Phys. Rev. Lett., 2008, 100, 118101(1-4).

20 T. Yoshidome and M. Kinoshita, Phys. Rev. E, 2009, 79, 090305(R)(1-4).

21 H. Oshima, T. Yoshidome, K. Amano, and M. Kinoshita, J. Chem. Phys., 2009, 131, 205102(1-11).

22 C. L. Dias, T. Ala-Nissila, J. Wong-ekkabut, I. Vattulainen, M. Grant, and M. Karttunen, Cryobiology, 2010, 60, 9199.

23 G. Graziano, Phys. Chem. Chem. Phys., 2010, 12, 1424514252.

24 M. Kinoshita and M. Harada, Mol. Phys., 1988, 65, 599618.

25 M. Kinoshita, S. Iba, K. Kuwamoto, and M. Harada, J. Chem. Phys., 1996, 105, 7177-7183.

26 M. Kinoshita, S. Iba, K. Kuwamoto, and M. Harada, J. Chem. Phys., 1996, 105, 7184-7191.

27 M. Kinoshita, J. Chem. Phys., 2003, 118, 8969-8981.

28 P. M. König, R. Roth, and K. R. Mecke, Phys. Rev. Lett., 2004, 93, 160601(1-4).

29 R. Roth, Y. Harano, and M. Kinoshita, Phys. Rev. Lett., 2006, 97, 078101(1-4).

30 R. Kodama, R. Roth, Y. Harano, and M. Kinoshita, J. Chem. Phys., 2011, 135, 045103(1-8).

31 N. Baden, S. Hirota, T. Takabe, N. Funasaki, and T. Terazima, J. Chem. Phys., 2007, 127, 175103(1-12).

32 T. Imai, Y. Harano, M. Kinoshita, A. Kovalenko, and F. Hirata, J. Chem. Phys., 2007, 126, 225102(1-9).

33 M. Kinoshita, Int. J. Mol. Sci., 2009, 10, 1064-1080.

34 M. Kinoshita, Front. Biosci., 2009, 14, 3419-3454.

35 T. Imai, Y. Harano, M. Kinoshita, A. Kovalenko, and F. Hirata, J. Chem. Phys., 2006, 125, 024911(1-7).

36 S. Yasuda, T. Yoshidome, H. Oshima, R. Kodama, Y. Harano, and M. Kinoshita, J. Chem. Phys., 2010, 132, 065105(1-10).

37 P. L. Privalov, Pure Appl. Chem., 2007, 79, 1445-1462.

38 J. Fitter, Biophys. J., 2003, 84, 3924-3930.

39 G. I. Makhatadze and P. L. Privalov, J. Mol. Biol., 1990, 213, 375-384.

40 K. P. Murphy and S. J. Gill, Thermochim. Acta, 1990, 172, 11-20.

41 G. Velicelebi and J. M. Sturtevant, Biochemistry, 1979, 18, 1180-1186.

42 P. L. Privalov and G. I. Makahatadze, J. Mol. Biol., 1990, 213, 385-391. 
43 J. M. Sturtevant, Proc. Natl. Acad. Sci. U.S.A., 1977, 74, 2236-2240.

44 M. Adrover, V. Esposito, G. Martorell, A. Pastore, and P. A. Temussi, J. Am. Chem. Soc., 2010, 132, 1624016246.

45 D. W. Rebertus, B. J. Berne, and D. Chandler, J. Chem. Phys., 1979, 170, 3395-3400.

46 T. Morita, Prog. Theor. Phys., 1960, 23, 829-845.

47 T. Morita and K. Hiroike, Prog. Theor. Phys., 1961, 25, 537-578.

48 T. Yoshidome, M. Kinoshita, S. Hirota, N. Baden and M. Terazima, J. Chem. Phys., 2008, 128, 225104(1-9).

49 H. S. Ashbaugh and M. E. Paulaitis, J. Phys. Chem., 1996, 100, 1900-1913.

50 Y. Harano, T. Yoshidome, and M. Kinoshita, J. Chem. Phys., 2008, 129, 145103(1-9).

51 T. Yoshidome, Y. Harano, and M. Kinoshita, Phys. Rev. E, 2009, 79, $011912(1-10)$

52 M. L. Connolly, J. Appl. Crystallogr., 1983, 16, 548-558.

53 M. L. Connolly, J. Am. Chem. Soc., 1985, 107, 1118-1124.

54 M. Ikeguchi and J. Doi, J. Chem. Phys., 1995, 103, 50115017.

55 M. Kinoshita, J. Chem. Phys., 2002, 116, 3493-3501.

56 K. Amano, T. Yoshidome, Y. Harano, K. Oda, and M. Kinoshita, Chem. Phys. Lett., 2009, 474, 190-194.

57 K. Oda, R. Kodama, T. Yoshidome, M. Yamanaka, Y. Sambongi, and M. Kinoshita, J. Chem. Phys., 2011, 134, 025101(1-9).

58 T. Yoshidome, Y. Ito, M. Ikeguchi, and M. Kinoshita, J. Am. Chem. Soc., 2011, 133, 4030-4039.

59 Y. Harano, R. Roth, Y. Sugita, M. Ikeguchi, and M. Kinoshita, Chem. Phys. Lett., 2007, 437, 112-116.

60 T. Yoshidome, K. Oda, Y. Harano, R. Roth, Y. Sugita, M. Ikeguchi, and M. Kinoshita, Proteins: Struct. Funct. Genet., 2009, 77, 950-961.

61 S. Yasuda, T. Yoshidome, Y. Harano, R. Roth, H. Oshima, K. Oda, Y. Sugita, M. Ikeguchi, and M. Kinoshita, Proteins: Struct. Funct. Genet., 2011, 79, 2161-2171.

62 M. Kinoshita, Y. Harano, and R. Akiyama, J. Chem. Phys., 2006, 125, 244504(1-7).

63 M. Ishizaki, H. Tanaka, and K. Koga, Phys. Chem. Chem. Phys., 2011, 13, 2328-2334.

64 K. Koga, Phys. Chem. Chem. Phys., 2011, 13, 1974919758.

65 T. V. Chalikian, and K. J. Breslauer, Biopolymers, 1996, 39, 619-626.

66 K. A. Dill, S. B. Ozkan, M. S. Shell, and T. R. Weikl, Annu. Rev. Biophys., 2008, 37, 289-316.

67 S. Asakura and F. Oosawa, J. Chem. Phys., 1954, 22,
1255-1256.

68 S. Asakura and F. Oosawa, J. Polym. Sci., 1958, 33, 183192.

69 Y. Harano and M. Kinoshita, Biophys. J., 2005, 89, 27012710.

70 J. R. Livingstone, R. S. Spolar, and M. T. Record Jr. Biochemistry, 1991, 30, 4237-4244.

71 N. Matubayasi and M. Nakahara, J. Chem. Phys., 2000, 112, 8089-8109.

72 H. Hansen-Goos, R. Roth, K. Mecke, and S. Dietrich, Phys. Rev. Lett., 2007, 99 128101(1-4). 
Table 1 Changes in solvation free energy $\Delta \mu$, contribution to free energy from conformational entropy of protein $-T \Delta S_{\mathrm{C}}$, and free energy of the entire system $\Delta G$ upon protein unfolding at $T=298.15$ $\mathrm{K}$ (in $\mathrm{kJ} / \mathrm{mol})$.

\begin{tabular}{cccc}
\hline & $\Delta \mu$ & $-T \Delta S_{\mathrm{C}}$ & $\Delta G$ \\
\hline Water & 479.1 & -429.1 & 50.0 \\
Simple solvent 1 & 622.3 & -429.1 & 193.2 \\
Simple solvent 2 & 204.5 & -429.1 & -224.6 \\
Hard-sphere solvent & 532.7 & -429.1 & 103.6 \\
\hline
\end{tabular}

Table 2 Changes in hydration free energy $\Delta \mu$ (i.e., free-energy changes of water) upon protein unfolding and in its terms 1 and 2 calculated at $T=258.15 \mathrm{~K}$ (in $\mathrm{kJ} / \mathrm{mol}$ ). The two values pertinent to $258.15 \mathrm{~K}\left(\rho_{\mathrm{S}} d_{\mathrm{S}}^{3}=0.7312\right)$ and $298.15 \mathrm{~K}\left(\rho_{\mathrm{S}} d_{\mathrm{S}}^{3}=0.7317\right)$, respectively, are employed for the number densities of water.

\begin{tabular}{lccc}
\hline & $\Delta \mu$ & Term 1 & Term 2 \\
\hline$\rho_{\mathrm{S}} d_{\mathrm{S}}^{3}=0.7312$ & 369.4 & 80.93 & 288.4 \\
$\rho_{\mathrm{S}} d_{\mathrm{S}}^{3}=0.7317$ & 370.7 & 82.18 & 288.5 \\
\hline
\end{tabular}




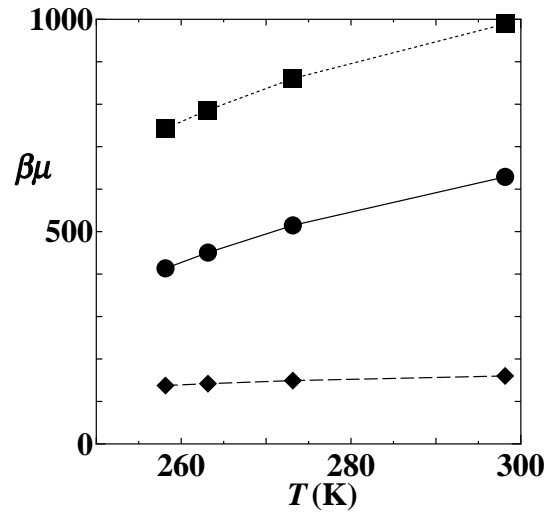

(a)

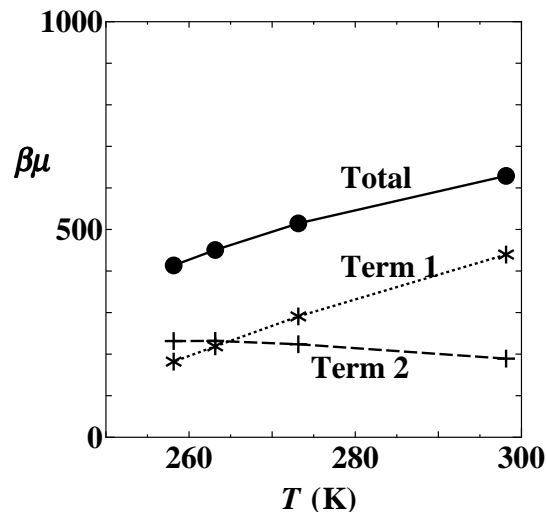

(c)

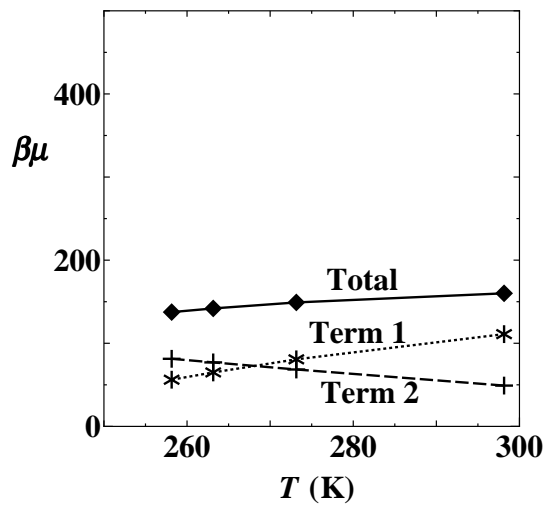

(e)

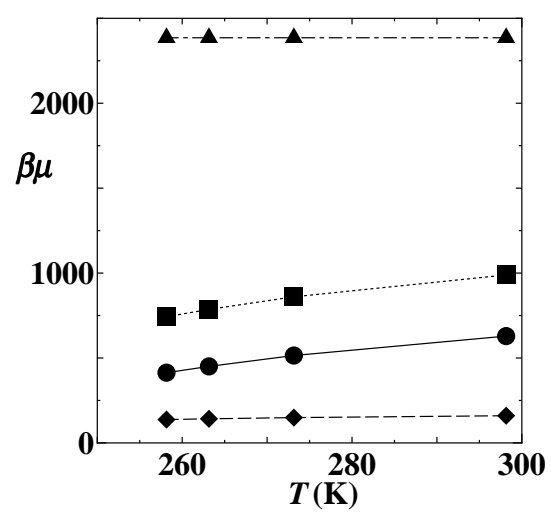

(b)

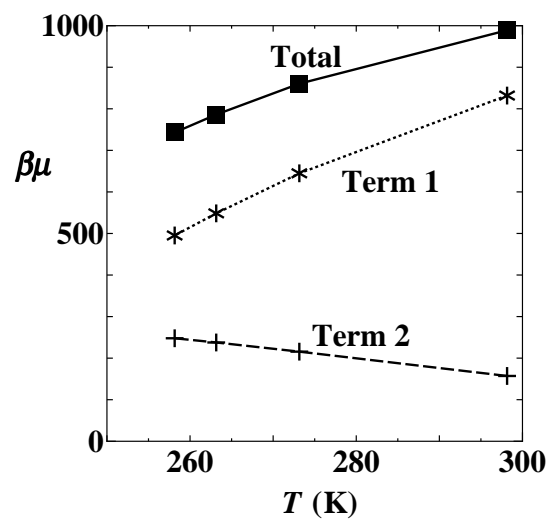

(d)

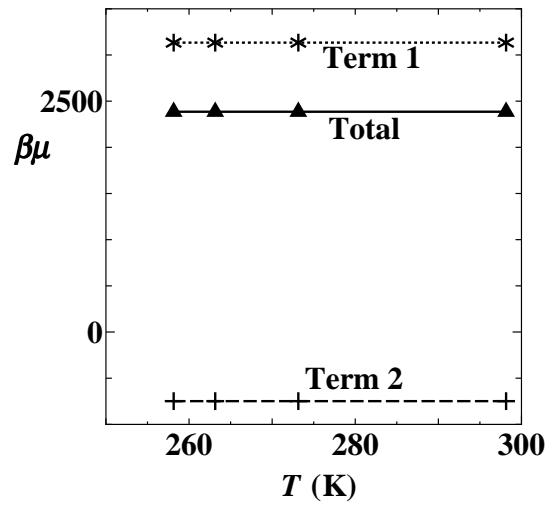

(f)

Fig. 1 (a) and (b): Temperature dependence of $\beta \mu$ of the native structure of protein $\mathrm{G}$ for water (circles), simple solvent 1 (squares), and simple solvent 2 (rhombuses). The result for the hard-sphere solvent (triangles) is given in (b). Temperature dependences of $\beta \mu$ and its terms 1 and 2 are shown for water (c), simple solvent 1 (d), simple solvent 2 (e), and the hard-sphere solvent (f). "Total" is the sum of terms 1 and 2 and equal to $\beta \mu$ itself. 


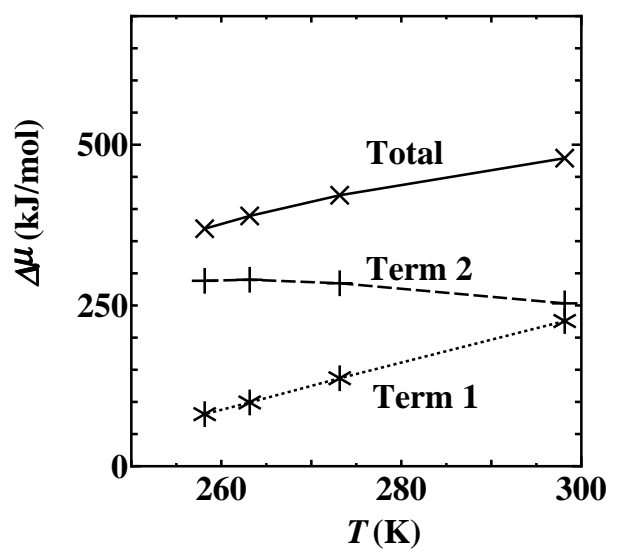

(a)

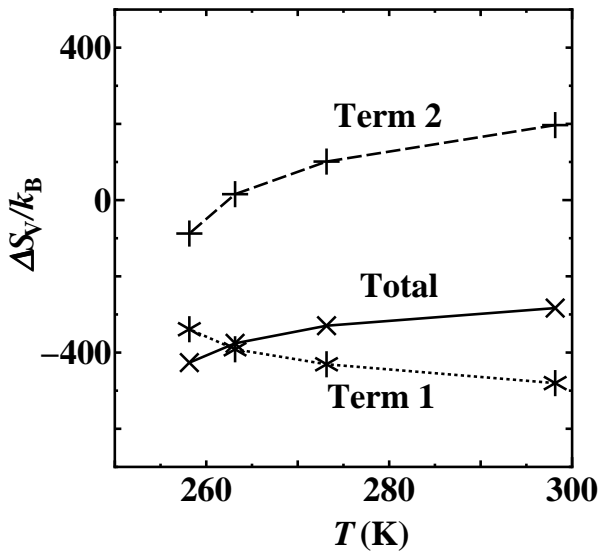

(c)

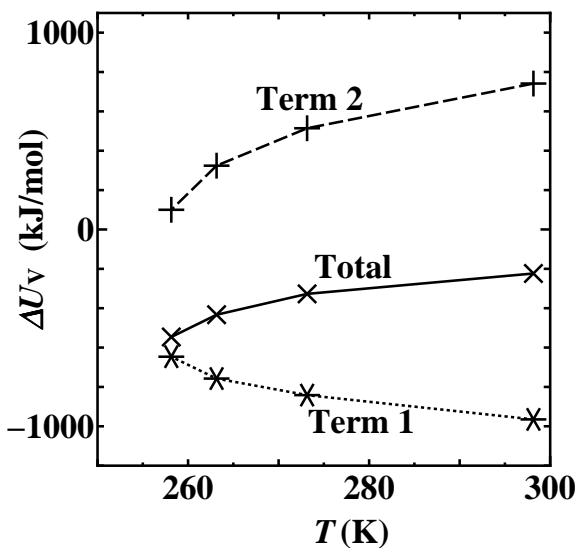

(b)

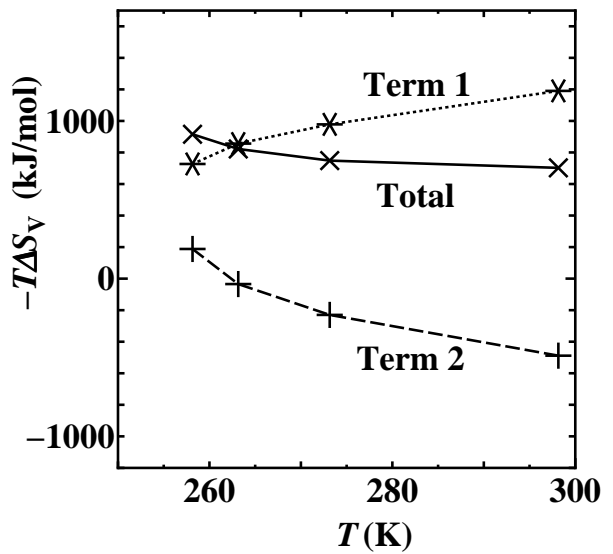

(d)

Fig. 2 Temperature dependences of changes in thermodynamic quantities of water upon protein unfolding: (a) free energy, (b) energy, (c) entropy, and (d) entropy multiplied by $-T$ (i.e., contribution to free energy from entropy). Term 1 is the first term in the right hand of Eq. (10) and term 2 is the sum of the other three terms. "Total" denotes the sum of terms 1 and 2. 


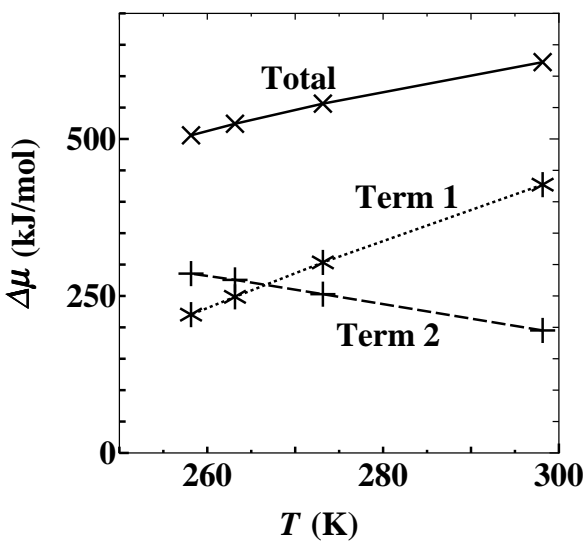

(a)

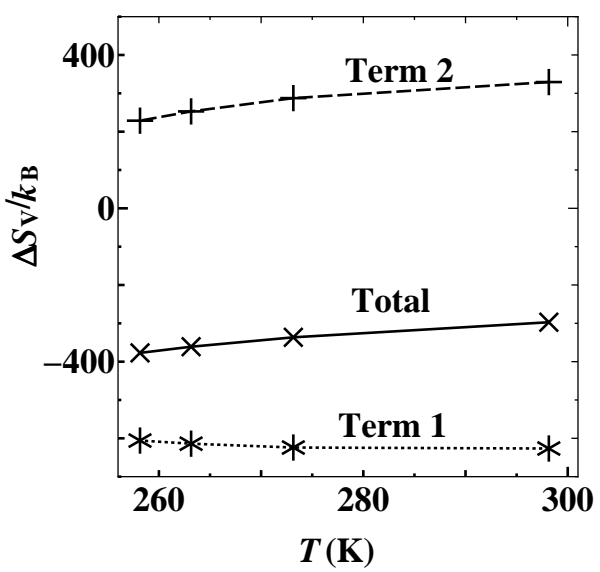

(c)

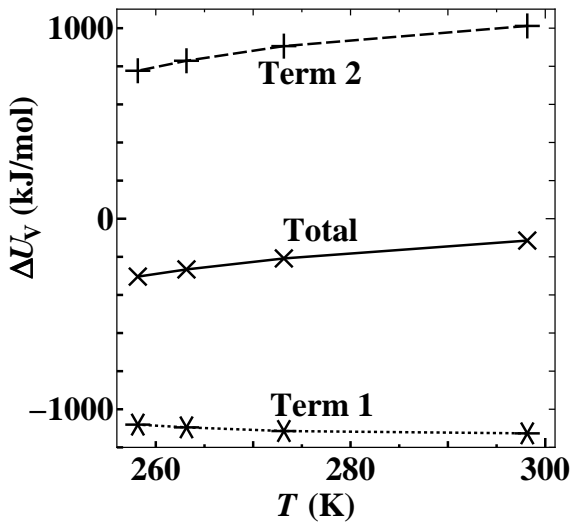

(b)

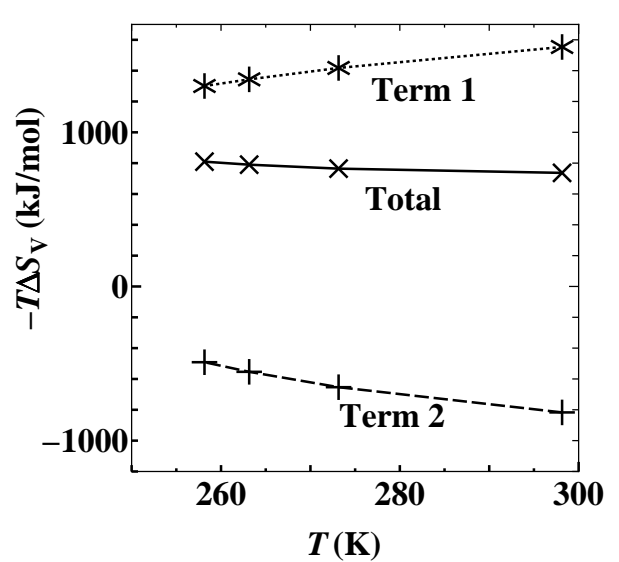

(d)

Fig. 3 Temperature dependences of changes in thermodynamic quantities of simple solvent 1 upon protein unfolding: (a) free energy, (b) energy, (c) entropy, and (d) entropy multiplied by $-T$ (i.e., contribution to free energy from entropy). Term 1 is the first term in the right hand of Eq. (10) and term 2 is the sum of the other three terms. "Total" is the sum of terms 1 and 2. 


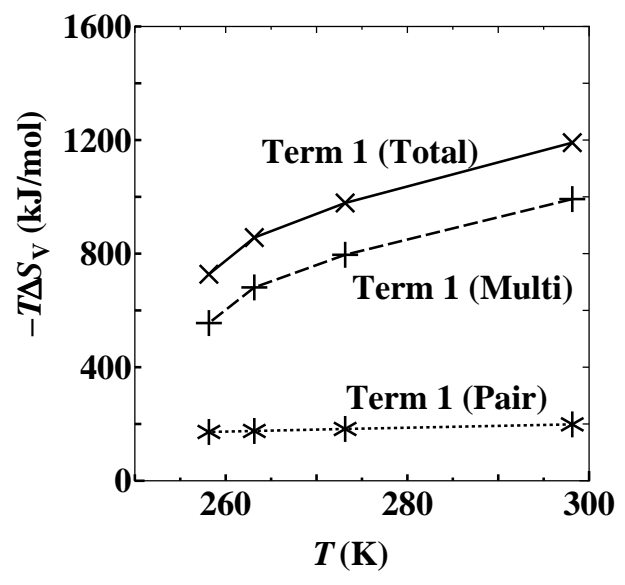

(a)

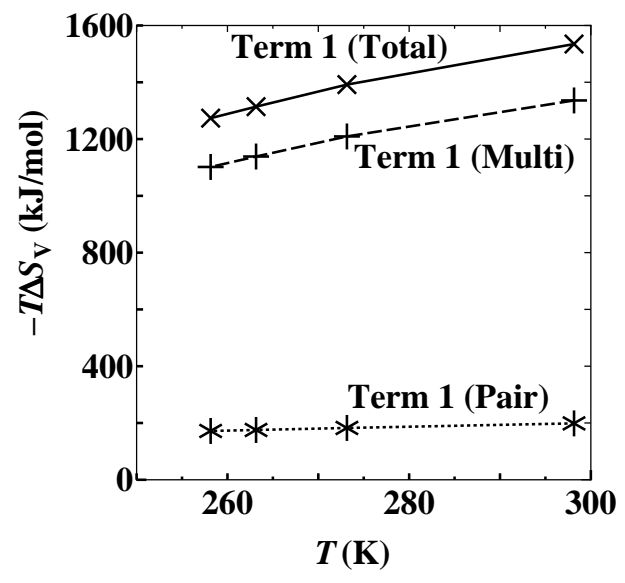

(b)

Fig. 4 Temperature dependences of term 1 of $-T \Delta S_{V}$ at the protein-solvent pair correlation level ["Term 1 (Pair)"] and that at the protein-solvent-solvent triplet and higher-order correlation level [“Term 1 (Multi)"]. The sum is indicated by "Term 1 (Total)". (a) Water. (b) Simple solvent 1. 


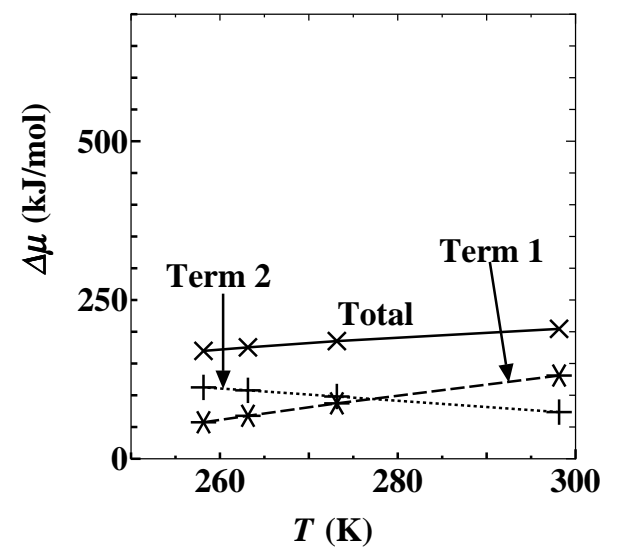

(a)

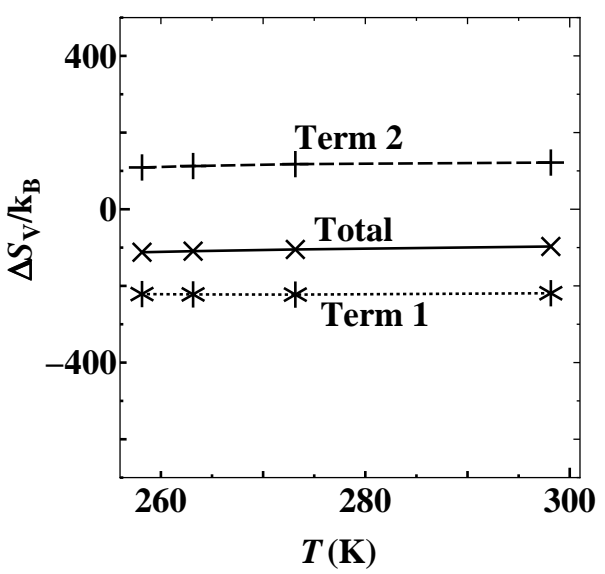

(c)

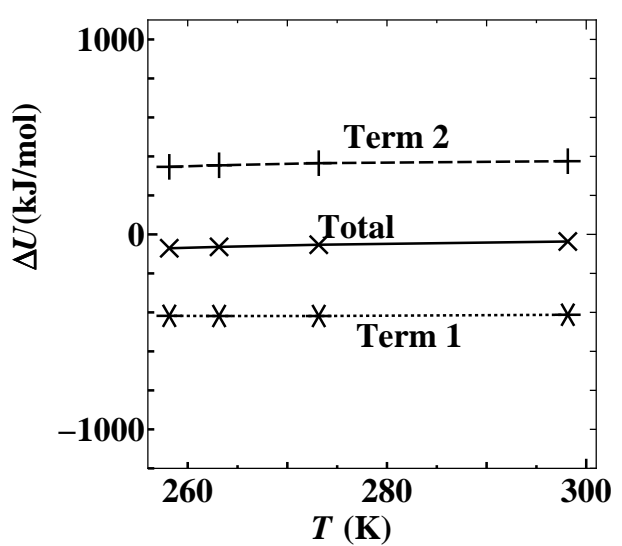

(b)

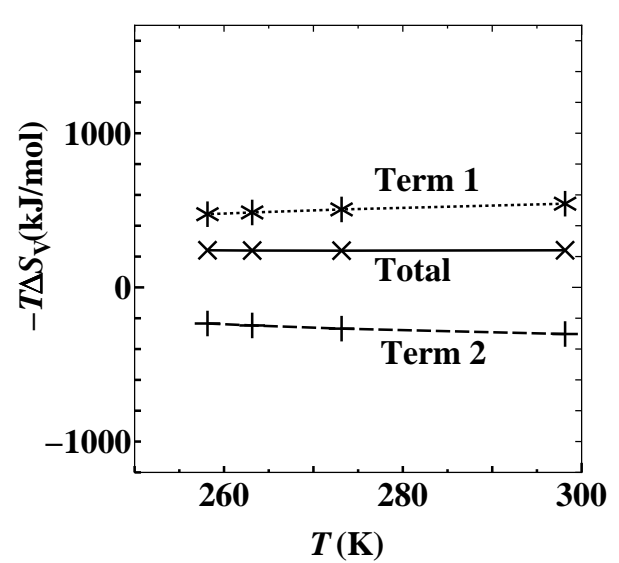

(d)

Fig. 5 Temperature dependences of changes in thermodynamic quantities of simple solvent 2 upon protein unfolding: (a) free energy, (b) energy, (c) entropy, and (d) entropy multiplied by $-T$ (i.e., contribution to free energy from entropy). Term 1 is the first term in the right hand of Eq. (10) and term 2 is the sum of the other three terms. "Total" is the sum of terms 1 and 2. 


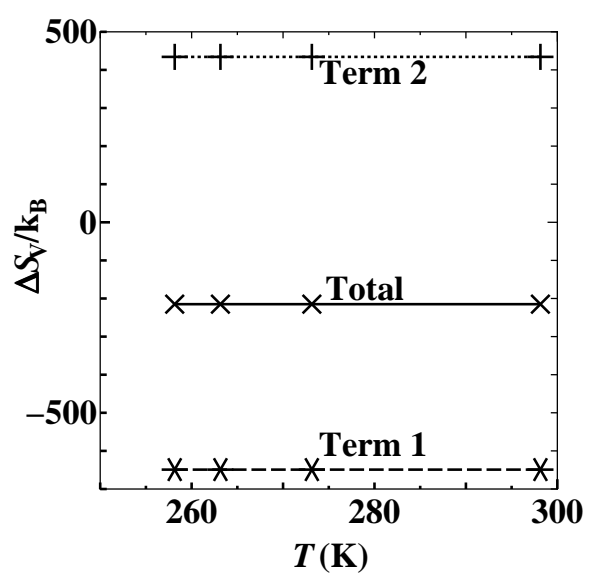

(a)

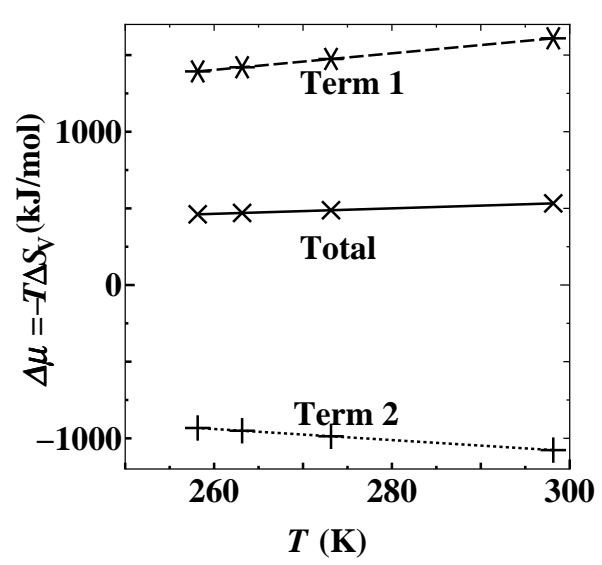

(b)

Fig. 6 Temperature dependences of changes in thermodynamic quantities of hard-sphere solvent upon protein unfolding: (a) entropy and (b) entropy multiplied by $-T$ corresponding to $\Delta \mu$. Term 1 is the first term in the right hand of Eq. (10) and term 2 is the sum of the other three terms. "Total" is the sum of terms 1 and 2. 


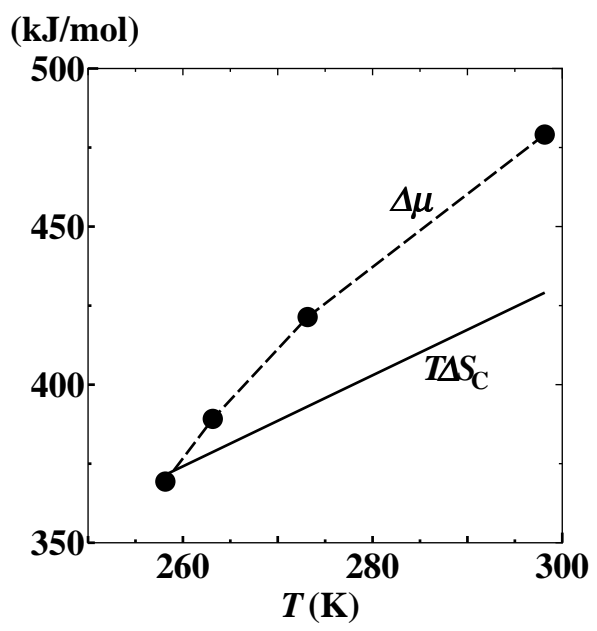

(a)

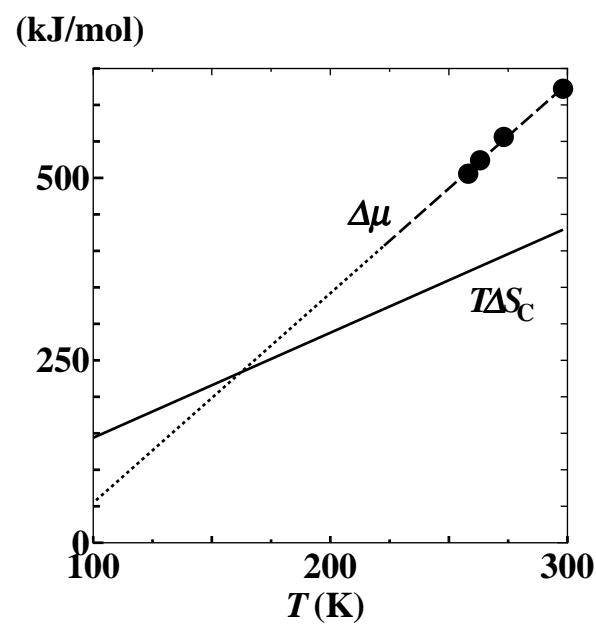

(b)

Fig. 7 Temperature dependences of changes in solvation free energy, $\Delta \mu$, and in protein conformational entropy multiplied by $T, T \Delta S_{\mathrm{C}}$. (a) Water. (b) Simple solvent 1 . The temperature at which the two lines intersect represents the cold denaturation temperature. In (b), we linearly extrapolate $\Delta \mu$ for temperatures lower than $258.15 \mathrm{~K}$. The dashed and dotted lines represent the extrapolated lines above and below the spinodal point $(\sim 222 \mathrm{~K})$, respectively. 